\title{
POINCARÉ-BENDIXSON THEORY FOR LEAVES OF CODIMENSION ONE
}

BY

\author{
JOHN CANTWELL ${ }^{1}$ AND LAWRENCE CONLON ${ }^{2}$
}

\begin{abstract}
The level of a local minimal set of a $C^{2}$ codimension-one foliation of a compact manifold is a nonnegative integer defined inductively, level zero corresponding to the minimal sets in the usual sense. Each leaf of a local minimal set at level $k$ is at level $k$. The authors develop a theory of local minimal sets, level, and how leaves at level $k$ asymptotically approach leaves at lower level. This last generalizes the classical Poincaré-Bendixson theorem and provides information relating growth, topological type, and level, e.g. if $L$ is a totally proper leaf at level $k$ then $L$ has exactly polynomial growth of degree $k$ and topological type $k-1$.
\end{abstract}

Introduction. Various authors have studied the extent to which the PoincaréBendixson theory of $C^{2}$-flows on closed surfaces [Sc] does or does not extend to $C^{2}$-foliations of codimension one on closed $n$-manifolds (e.g., cf. [P2], [P3], [P5], [Sa1], [Sa2], [C-C3], [M-P]). We present here a systematic treatment of the limit set of an arbitrary leaf in such foliations, producing new results of the above type and, we hope, providing a helpful overall perspective on previous results. We wish to acknowledge the fundamental dependence of this paper on ideas of A. J. Schwartz [Sc], R. Sacksteder [Sa2], J. F. Plante [P1], [P4], and G. Hector (thesis). We wish also to acknowledge that the results presented here are known independently by $G$. Hector and G. Duminy and that extensive conversations with them have helped us all to reach a uniform terminology and points of view that are not totally dissimilar.

Conventions. Throughout this paper, $M$ will be a closed, orientable $n$-manifold and $\mathscr{F}$ will be a transversely orientable $C^{2}$-foliation of $M$ by leaves of dimension $n-1$.

The limit set of a leaf will be analyzed in terms of the local minimal sets that it contains (also called relative minimal sets [Di]). A local minimal set is a minimal set of $\mathscr{F} \mid U$, where $U$ is some open, $\mathscr{F}$-saturated set. The level of a local minimal set will be a nonnegative integer, defined inductively (cf. $§ 1(D))$, level zero corresponding to the minimal sets of $\mathscr{F}$ in the usual sense. Each leaf of a local minimal set at level $k$ is itself said to be at level $k$. Leaves that lie in no local minimal set have limit sets containing leaves at each finite level; hence they are said to be at infinite

Received by the editors November 29, 1979; presented to the Society, January 4, 1978.

1980 Mathematics Subject Classification. Primary 57R30; Secondary 58F21, 58H05.

Key words and phrases. Poincaré-Bendixson theorem, local minimal set, level, infinite level, totally proper leaf, limit set, growth, end, pseudogroup, biregular cover.

${ }^{1}$ Research partially supported by NSF grant MCS 77-01411.

${ }^{2}$ Research partially supported by NSF grant MCS 77-01418. 
level. Such leaves can exist [He], [C-C3], [C-C5], even in $C^{\infty}$-foliations, and they exhibit rather wild behavior. Smooth-leaved $C^{0}$-foliations can be constructed that contain a whole infinite hierarchy of infinite levels, but $C^{2}$-foliations admit at most one such level.

Of particular interest in this theory are the proper leaves of $\mathscr{F}$. Every proper leaf is a local minimal set. If its limit set consists only of proper leaves, the proper leaf will be said to be totally proper. Such leaves play a basic role in the theory of leaves with nonexponential growth and themselves display growth properties and topological properties rigidly associated to their level. These properties follow easily from the Poincaré-Bendixson theory of totally proper leaves (\$6) in which the way that such leaves "spiral in" on leaves at lower levels is explicitly described.

The condition that $\mathscr{F}$ be of class at least $C^{2}$ is essential to most of this paper. Examples show that a Poincaré-Bendixson theory for $C^{1}$-foliations will be considerably more complicated (e.g., [C-C1, (4.3)], [De], [Sch, Appendix]). The sources of this dependence on $C^{2}$-differentiablity are the technical results on pseudogroups collected in $\S 2$.

Statements of results. We collect here the principal theorems ( $m .0)$ of each of the sections $m=3,4,5,6,7$. Definitions of the technical terms used will be found in $\S 1$.

The first result is a fundamental existence theorem for local minimal sets. It seems to be contained in Hector's thesis, but, as of this writing, a proof is not to be found in the available literature.

(3.0) ThEOREM (HeCtOR). Let $U \subseteq M$ be an open, connected, $\mathcal{F}$-saturated set and let $L \subset U$ be a leaf of $\mathcal{F}$. Then $\bar{L} \cap U$ contains a minimal set of $\mathcal{F} \mid U$.

Let $M_{k}$ denote the union of all leaves at levels at most $k$. Let $C_{k}$ denote the union of all totally proper leaves at levels at most $k$.

(4.0) THEOREM. Each local minimal set lies at some finite level $k$. For each integer $k \geqslant 0, M_{k}$ and $C_{k}$ are compact sets. Finally, if $L$ is an arbitrary leaf of $\mathscr{F}$ and $k>0$ is an arbitrary integer, then $\bar{L} \cap M_{k}$ is a finite (nonempty) union of local minimal sets; hence $\bar{L} \cap C_{k}$ is a finite (possibly empty) union of leaves.

If there are any leaves in $M-\cup_{k>0} M_{k}$, they are said to be at infinite level. Part (a) of the following theorem justifies this terminology. The symbol $S(L)$ stands for the substructure of $L(\S 1)$, which, in this case, is the union of all leaves of $\bar{L}$ that are at finite level.

(5.0) TheOREM. Let $\mathcal{F}$ be of class $C^{r}, 2 \leqslant r \leqslant \infty$, and let $L$ be a leaf at infinite level. Let $Z=\bar{L}-S(L)$. Then

(a) for each integer $k \geqslant 0, S(L)$ contains at least one leaf at level $k$, and either all of the leaves of $S(L)$ or only finitely many of them are totally proper;

(b) the set $S(L)$ is dense in $\bar{L}$, but it contains no leaf that is dense in $\bar{L}$;

(c) the set $Z$ is an uncountable union of leaves, each of which is dense in $\bar{L}$;

(d) no leaf of $Z$ has a proper side;

(e) the holonomy of each leaf of $Z$ is $C^{r}$-tangent to the identity. 
REMARK. If $L$ is at infinite level and if $L_{0}$ is a leaf asymptotic to $L$, then $L_{0}$ is also at infinite level (4.0) and $\bar{L}_{0}=S\left(L_{0}\right) \cup Z_{0}$ as in (5.0), with $L \subset Z_{0}$. By (5.0), part (c), $L$ is asymptotic to $L_{0}$, so $\bar{L}_{0}=\bar{L}, S\left(L_{0}\right)=S(L)$, and $Z_{0}=Z$. In particular, this justifies our earlier remark that there can only be one infinite level.

The results in $\$ 6$ give a generalization, for totally proper leaves, of the familiar Poincaré-Bendixson phenomenon of a flow line (on a surface) winding in on a periodic orbit. It would be clumsy to formulate this here, but we do formulate the following striking consequence (announced by the authors in [C-CO] and also obtained more recently by $\mathrm{N}$. Tsuchiya [Ts]).

(6.0) THEOREM. The totally proper leaves of $\mathscr{F}$ are precisely the nowhere dense leaves with growth dominated by a polynomial. If the totally proper leaf $L$ is at level $k$, then it has exactly polynomial growth of degree $k$ and $L$ is a manifold of type $k-1$.

For the definitions of "exactly polynomial growth" and of "manifolds of type $k-1$ ", see $\$ 1$.

In [C-C4, $\S \S 1$ and 2], the results of the present paper were reviewed and in $\S 3$ of the same reference certain corollaries were drawn that can be assembled into the following theorem. Here, of course, to say that $L$ has totally proper substructure means that $S(L)$ is a union of totally proper leaves.

(7.0) THEOREM. If $L$ is a leaf with nonexponential growth, then $L$ has totally proper substructure. If, in addition $L$ is at finite level, then either

(a) $L$ is a totally proper leaf; or

(b) the set $U=\bar{L}-S(L)$ is an open, local minimal set, $\mathscr{F} \mid U$ has trivial holonomy, the leaves of $\mathscr{F} \mid U$ are mutually diffeomorphic manifolds of finite type, and these leaves have the same growth as $L$.

In $\S 7$, we will draw some corollaries to (7.0) that were not noted in [C-C4].

\section{Review of technical concepts.}

(A) Biregular covers. Fix a smooth, one-dimensional foliation $\mathcal{L}$ transverse to $\mathscr{F}$. Fix a transverse orientation for $\mathscr{F}$, inducing thereby an orientation on each leaf of $\mathcal{L}$. If $x$ and $y$ lie in a common leaf $R$ of $\mathcal{L}$ and $x<y$ in the orientation of $R$, then denote the closed subarc from $x$ to $y$ by $[x, y]$. Of course, $R$ might be a closed leaf, in which case also $y<x$ and $R=[x, y] \cup[y, x]$. Similarly, we will consider the subarcs $(x, y),(x, y]$, and $[x, y)$. If subarcs are parametrized by real numbers, this parametrization will be chosen to respect orientation.

Let $D^{n-1} \subset \mathbf{R}^{n-1}$ denote the closed unit disk and let $I=[-1,1]$. An $(\mathscr{F}, \mathcal{L})$ coordinate chart $(W, \varphi)$ on $M$ is an imbedding $\varphi: D^{n-1} \times I \rightarrow M, W=\operatorname{Im}(\varphi)$, such that $\varphi \mid\left(D^{n-1} \times\{t\}\right)$ is an imbedding into a leaf of $\mathscr{F}, \forall t \in I$, and $\varphi \mid(\{x\} \times I)$ is an imbedding into a leaf of $\mathcal{L}, \forall x \in D^{n-1}$. We fix a finite atlas $\left\{\left(W_{i}, \varphi_{i}\right)\right\}_{i=1}^{m}$ of $(\mathscr{F}, \mathcal{L})$ coordinate charts on $M$ such that $\left\{\operatorname{int}\left(W_{i}\right)\right\}_{i=1}^{m}$ is an open cover of $\boldsymbol{M}$. 
Definition. The atlas $\left\{\left(W_{i}, \varphi_{i}\right)\right\}_{i=1}^{m}$ is called a biregular cover of $M$ provided that, whenever $W_{i} \cap W_{j} \neq \varnothing$, then $W_{i} \cup W_{j} \subseteq \operatorname{int}(W)$, where $(W, \varphi)$ is an $(\mathcal{F}, \mathcal{L})$ coordinate chart on $\boldsymbol{M}$.

It is elementary that biregular covers exist.

The holonomy pseudogroup $\Gamma_{\mathscr{F}}$ of $\mathscr{F}$ is defined on the disjoint union of all leaves of $\mathcal{L}$, the domain and range of each $\gamma \in \Gamma_{\mathscr{F}}$ being a finite union of (not necessarily open) subarcs. Each $\gamma \in \Gamma_{\mathscr{F}}$ will be understood to be defined using only "fences" of the form $f(t, s)$ where, for each fixed $t, f_{t}=f(t, \cdot)$ takes its image in a leaf of $\mathcal{E}$, and, for each fixed $s, f_{s}=f(\cdot, s)$ takes its image in a leaf of $\mathcal{F}$.

Given a biregular cover $\left\{\left(W_{i}, \varphi_{i}\right)\right\}_{i=1}^{m}$, a subpseudogroup $\Gamma \subset \Gamma_{\mathscr{F}}$ finitely generated and containing all of the information in $\Gamma_{\mathscr{F}}$ is defined as follows. Let $R_{i}=\varphi_{i}(\{0\} \times I), 1 \leqslant i \leqslant m$, and let $R$ be the disjoint union of these subarcs. If $W_{i} \cap W_{j} \neq \varnothing$, there are holonomy transition functions $\gamma_{i j}: R_{i j} \rightarrow R_{j i}$ where $R_{i j} \subseteq$ $R_{i}$ and $R_{j i} \subseteq R_{j}$ are the maximal possible range and domain respectively. Of course, $\gamma_{j i}: R_{j i} \rightarrow R_{i j}$ is the inverse of $\gamma_{i j}$. The pseudogroup $\Gamma$ is the pseudogroup on $R$ generated by these transition functions.

(B) Growth. Fix a biregular cover $\left\{\left(W_{i}, \varphi_{i}\right)\right\}_{i=1}^{m}$ of $M$. Given a leaf $L$ of $\mathscr{F}$, fix a plaque $P_{0}=\varphi_{i}\left(D^{n-1} \times\left\{t_{0}\right\}\right) \subset L$. There corresponds a growth function $g_{L}: \mathbf{Z}^{+}$ $\rightarrow \mathrm{Z}^{+}$for $L$ defined by letting $g_{L}(m)$ be the number of plaques $P_{*}$ that can be reached by chains of plaques $P_{0}, P_{1}, \ldots, P_{r}=P_{*}$, where $r<m$ and $P_{i} \cap P_{i-1} \neq$ $\varnothing, 1 \leqslant i \leqslant r$. It will be convenient to consider $g_{L}$ as a function into $\mathbf{R}^{+}$. Evidently $g_{L}$ is nondecreasing.

Definition. Let $f, h: \mathbf{Z}^{+} \rightarrow \mathbf{R}^{+}$be nondecreasing. If there are positive constants $A, B, C$ such that $f(t) \leqslant A h(B t), \forall t \geqslant C$, then $h$ is said to dominate $f$. If $f$ and $h$ dominate each other, they are said to have the same growth type. The growth type of $f$ is denoted $\operatorname{gr}(f)$.

Mutual dominance is an equivalence relation and $\operatorname{gr}(f)$ is the equivalence class of $f$. It is a standard consequence of the compactness of $M$ that $\operatorname{gr}\left(g_{L}\right)$ depends only on the leaf $L$, not on the choices of biregular cover and of base plaque $P_{0}$.

Definition. The growth type of the leaf $L$ is $\operatorname{gr}(L)=\operatorname{gr}\left(g_{L}\right)$. In particular, $L$ has exactly polynomial growth of degree $k$ if $\operatorname{gr}\left(g_{L}\right)$ contains the polynomial $f(m)=$ $m^{k}$. The leaf $L$ has exponential growth if $\operatorname{gr}\left(g_{L}\right)$ contains the function $f(m)=e^{m}$.

It is common to define polynomial growth of degree $k$ to mean that $m^{k}$ dominates $g_{L}$, hence the word "exactly" in the above definition. It is also common to define exponential growth to mean that $g_{L}$ dominates $e^{m}$. But compactness of $M$ implies that $e^{m}$ dominates $g_{L}$, so the following (standard) definition of exponential growth coincides with the one given above.

Definition. The leaf $L$ has exponential growth if $\lim _{\inf _{m \rightarrow \infty}}(1 / m) \log \left(g_{L}(m)\right) \neq$ 0 . Otherwise, $L$ has nonexponential growth.

(C) Limit sets and ends. Let $L$ be a leaf and let $\left\{K_{\alpha}\right\}_{\alpha \in A}$ denote the family of all compact subsets of $L$. Let $W_{\alpha}=L-K_{\alpha}$. As usual, $W_{\alpha}$ denotes closure in $M$.

Definition. The limit set of $L$ is the set $\lim (L)=\bigcap_{\alpha \in A} \bar{W}_{\alpha}$.

It is elementary that $\lim (L)$ is compact and $\mathscr{F}$-saturated. If $L$ is noncompact, then $\lim (L)$ is nonempty. If $L^{\prime} \subset \lim (L)$ is a leaf, we say that $L$ is asymptotic to $L^{\prime}$ (or, approaches $L^{\prime}$ ). 
If $L$ is noncompact, it is possible to choose systems $\left\{U_{\alpha}\right\}_{\alpha \in A}$ such that each $U_{\alpha}$ is an (unbounded) component of $W_{\alpha}$ and such that each finite intersection $U_{\alpha_{1}} \cap \cdots \cap U_{\alpha_{q}}$ is nonempty (and unbounded). Then $\left\{U_{\alpha}\right\}_{\alpha \in A}$ is said to define an end $e$ of $L$ and to be the open neighborhood system of $e$ in $L$.

DEFINITION. The $e$-limit set of $L$ is

$$
e-\lim (L)=\bigcap_{\alpha \in A} \bar{U}_{\alpha}
$$

Evidently $e-\lim (L)$ is a nonempty, compact, $\mathscr{F}$-saturated subset of $\lim (L)$. If $L^{\prime} \subset e-\lim (L)$ is a leaf, we say that $e$ is asymptotic to $L^{\prime}$ (or, approaches $L^{\prime}$ ).

Definition. An end $e$ of $L$ is proper if $L \nsubseteq e-\lim (L)$. The end $e$ is totally proper if $e-\lim (L)$ is a union of proper leaves.

We will denote by $\mathcal{E}(L)$ the set of ends of $L$. A sequence $\left\{x_{m}\right\}$ in $L$ is said to converge to $e \in \mathcal{E}(L)$ if each neighborhood $U_{\alpha} \subseteq L$ of $e$ contains all but finitely many terms of the sequence. Similarly, a sequence $\left\{e_{m}\right\}$ in $\mathscr{E}(L)$ converges to $e \in \mathcal{E}(L)$ if each neighborhood $U_{\alpha} \subset L$ of $e$ is also a neighborhood of all but finitely many terms of the sequence. All of this is defined without reference to the foliation and provides a compact, metrizable topology on $L \cup \mathcal{E}(L)$ in which $\mathscr{E}(L)$ is a compact, totally disconnected subspace and the open subset $L$ has its intrinsic manifold topology (for details, see [A-S]; for some intuitive examples, see [C-C2] and [C-C3]).

As in [C-C3], we define, for each ordinal $\gamma \geqslant 0$, the $\gamma$ th derived set $\mathcal{E}^{(\gamma)}(L)$. Here $\mathcal{E}^{(0)}(L)=\mathcal{E}(L), \mathcal{E}^{(\gamma+1)}(L)$ is the set of cluster points in $\mathcal{E}^{(\gamma)}(L)$, and, if $\gamma$ is a limit ordinal, $\mathcal{E}^{(\gamma)}(L)=\cap_{\beta<\gamma} \mathcal{E}^{(\beta)}(L)$. Either there is a countable ordinal $\gamma$ such that $\mathcal{E}^{(\gamma)}(L)$ is a finite, nonempty set, or $\mathcal{E}^{(\Omega)}(L)$ is the Cantor set, where $\Omega$ denotes the first uncountable ordinal.

Definition. If $\mathcal{E}^{(\gamma)}(L)$ is a finite, nonempty set, $L$ has (topological) type $\gamma$. If $\mathcal{E}^{(\Omega)}(L)$ is a Cantor set, $L$ has type $\Omega$. If $L$ is compact, it is said to have type -1 .

Definition. An end $e \in \mathcal{E}(L)$ is said to be of type $\gamma<\Omega$ if $e$ is an isolated point in $\mathcal{E}^{(\gamma)}(L)$. If $e \in \mathcal{E}^{(\Omega)}(L)$, then $e$ has type $\Omega$.

(D) Local minimal sets and levels. Let $U \subseteq M$ be an open, $\mathscr{F}$-saturated set. A minimal set of the foliation $\mathscr{F} \mid U$ is said to be a local minimal set of $\mathscr{F}$. For smooth-leaved $C^{0}$-foliations, such minimal sets need not exist, but (3.0) guarantees their existence for $C^{2}$-foliations.

It is convenient to formulate the following more intrinsic definition.

Definition. Let $X \subseteq M$ be a nonempty, $\mathscr{F}$-saturated set. If $\bar{X}-X$ is compact and if each leaf of $X$ is dense in $\bar{X}$, then $X$ will be called a local minimal set of $\mathscr{F}$.

REMARK. If $X$ is as above and if $U$ denotes the open, $\mathscr{F}$-saturated set $M-(\bar{X}-$ $X$ ), then $X \subseteq U$ is a minimal set of $\mathscr{F} \mid U$. Conversely, if $U$ is an arbitrary, open, $\mathscr{F}$-saturated set and if $X \subseteq U$ is a minimal set of $\mathscr{F} \mid U$, then $X$ satisfies the above definition.

There are three types of local minimal sets:

(a) every proper leaf is such a set;

(b) a connected, open, $\mathscr{F}$-saturated subset $U \subseteq M$, in which each leaf of $\mathscr{F} \mid U$ is dense in $U$, is a local minimal set of locally dense type; 
(c) a local minimal set $X$ of neither type (a) nor (b) is said to be of exceptional type.

In type (c), $X$ is nowhere dense and meets each transverse, open arc in a set homeomorphic to an open subset of the Cantor set.

Evidently, a local minimal set $X$ either fails to meet a given compact, $\mathscr{F}$ saturated set $Y$, or $X \subseteq Y$. Consequently, two local minimal sets either coincide or are disjoint.

Definition. A minimal set of $\mathscr{F}$, and each of its leaves, is said to be at level 0 . A local minimal set $X$, and each of its leaves, is at level $k>0$ if $\bar{X}-X$ consists entirely of leaves at levels at most $k-1$, at least one of which is at level $k-1$.

Definition. An end $e$ of a leaf $L$ is at level $k \geqslant 0$ if $e-\lim (L)$ is a union of leaves at levels at most $k$, at least one of which is at level $k$.

ExAmple. Foliate $T^{3}$ by dense, planar leaves in standard fashion. Along a closed transversal to this foliation, perform the standard modification that inserts a Reeb component. The family of locally dense leaves constitutes a local minimal set of $U$ of locally dense type at level 1 . Each of these leaves has a countable set of isolated ends, each asymptotic only to the toral leaf; hence these ends are totally proper at level 0 . There is also an end $e$ of such a leaf $L \subset U$ asymptotic to all leaves in $U$, and this end is at level 1.

We will see (\$6) that totally proper ends at level $k$ are of type $k$.

Definition. A leaf belonging to no local minimal set is said to be at infinite level.

Definition. If $L$ is a leaf of $\mathscr{F}$, the substructure $S(L)$ is the union of all leaves of $\bar{L}$ at levels strictly less than the level of $L$.

(E) A convention. Throughout this paper, we will only use the symbol $\subset$ when equality is excluded, and the symbol $\subseteq$ when equality is allowed.

2. Pseudogroups of $C^{2}$-diffeomorphisms. Let $R \subseteq \mathbf{R}$ be a one-dimensional submanifold, not necessarily connected and possibly having $\partial R \neq \varnothing$. Let $\Gamma$ be a pseudogroup on $R$ generated by a finite family $\Gamma_{0}$ of local, orientation preserving $C^{2}$-diffeomorphisms. As in [P1], we do not require that the elements of the pseudogroup all have open domains, but it seems reasonable to exclude highly pathological domains by allowing only sets that are finite unions of intervals, whether open, closed, or half-open.

(A) $A$ theorem of Sacksteder. Let $C \subset \operatorname{int}(R)$ be a $\Gamma$-invariant Cantor set such that the interior of the domain of each element of $\Gamma_{0}$ has compact intersection with $C$. The proof of Theorem 1 of [Sa2] establishes the following.

(2.1) Theorem. Let $q \in C$ be an endpoint of a gap of $C$ and suppose that the $\Gamma$-orbit $\Gamma(q)$ clusters at $q$. Then there is a compact, connected neighborhood $U$ of $q$ in $R$ and a sequence $\left\{g_{m}\right\} \subset \Gamma$ such that

(1) $U \subseteq \operatorname{dom}\left(g_{m}\right), \forall m$;

(2) $\lim _{m \rightarrow \infty} g_{m}(q)=q$;

(3) $\lim _{m \rightarrow \infty} g_{m}^{\prime}(t)=0$ uniformly for $t \in U$. 
(2.2) Corollary. If $q, U$, and $\left\{g_{m}\right\}$ are as in (2.1) and if $V \subseteq U$ is a compact neighborhood of $q$, there is an integer $N \geqslant 0$ such that, for each $m \geqslant N, g_{m}$ maps $V$ into itself and has a unique fixed point $x_{m} \in V$. Indeed, $x_{m} \in C$ and $g_{m}$ is a 2-sided contraction at $x_{m}$.

This corollary is an easy consequence of $(2.1)$ and the contraction lemma [B, p. 43].

Here is another consequence of (2.1).

(2.3) Corollary. Let $\Gamma, C$, and $q \in C$ be as in (2.1). Let $\left\{q_{n}\right\} \subset R$ be a sequence converging to $q$. Then, for $n$ sufficiently large, $q$ is an element of the closure of the orbit $\Gamma\left(q_{n}\right)$.

Proof. Otherwise, the closure of $\Gamma\left(q_{n}\right)$ has a closest point $p_{n}$ to $q, p_{n} \neq q$, for each $n$. We can assume that $\left\{p_{n}\right\}$ converges strictly monotonically to $q$. For $n$ sufficiently large, there are compact neighborhoods $V_{n}$ and $V_{n+1}$ of $q$ such that $V_{n+1} \subset V_{n} \subset U, V_{n} \cap \Gamma\left(p_{n}\right)=p_{n}$, and $V_{n+1} \cap \Gamma\left(p_{n+1}\right)=p_{n+1}$. We can assume $m$ large enough that $g_{m}\left(V_{n}\right) \subset V_{n}, g_{m}\left(V_{n+1}\right) \subset V_{n+1}$, and that $g_{m}$ has a unique fixed point in $V_{n}$. But our conditions imply that $g_{m}\left(p_{n}\right)=p_{n}$ and $g_{m}\left(p_{n+1}\right)=p_{n+1}$, and that $p_{n}$ and $p_{n+1}$ are distinct points of $V_{n}$, a contradiction.

REMARK. In applying (2.1) and its corollaries to foliations, we will regularly encounter the following situation. There will be a nonproper leaf $L$ with a proper side, and a compact, transverse arc $\tilde{R}$ properly crossing $L$ in a point $q$. The endpoints of $\tilde{R}$ can be chosen to miss $\bar{L}$; hence $\tilde{R} \cap \bar{L}=\tilde{C}$ is a Cantor set. There is a pseudogroup $\tilde{\Gamma}$ on $\tilde{R}$ generated by the holonomy along the leaves. Clearly, $q$ will be an endpoint of a gap, $\tilde{\Gamma}(q)$ will cluster at $q$, and $\tilde{C}$ is $\tilde{\Gamma}$-invariant. The problem is that $\tilde{\Gamma}$ may not be generated by a finite subset $\left\{\gamma_{1}, \ldots, \gamma_{r}\right\}$ such that $\tilde{C} \cap \operatorname{int}\left(\operatorname{dom}\left(\gamma_{i}\right)\right)$ is compact, $1 \leqslant i \leqslant r$. This difficulty is dealt with as follows. Choose a biregular cover $\left\{\left(W_{i}, \varphi_{i}\right)\right\}_{i=1}^{r}$ such that $\varphi_{i}\left(D^{n-1} \times\{ \pm 1\}\right)$ does not meet any leaf of $\bar{L}, 1 \leqslant i \leqslant r$. As in $\S 1(\mathrm{~A})$, define the holonomy pseudogroup $\Gamma$ on the disjoint union $R=\cup_{i=1}^{r} R_{i}$, finitely generated by the transition functions $\gamma_{i j}: R_{i j}$ $\rightarrow R_{j i}, 1 \leqslant i, j \leqslant r$. Without loss of generality, assume $R_{1}=\tilde{R}$. Then $C=\bar{L} \cap R$ is a holonomy-invariant Cantor set, and $C \cap \operatorname{int}\left(R_{i j}\right)$ is compact, $1<i, j \leqslant r$. One then applies (2.1) and its corollaries to $R, C, q$, and $\Gamma$.

(B) Permuter pseudogroups. Let $\mathcal{G}$ be a collection of compact subintervals of $R$ with disjoint interiors. Also, assume that $R$ is compact.

Definition. Let $g \in \Gamma$. We will say that $g$ is a permuter of $g$ if $\operatorname{dom}(g)$ is compact and if, for each $J \in \mathcal{G}$, either int $(J) \cap \operatorname{dom}(g)=\varnothing$ or $J \subseteq \operatorname{dom}(g)$ and $g(J) \in g$.

Definition. If $\Gamma$ admits a finite, symmetric generating set $\Gamma_{0}$ consisting of permuters of $\mathcal{G}$, we will say that $\Gamma$ is a finitely generated permuter pseudogroup of g.

Fix the hypothesis that $\Gamma$ is a finitely generated permuter pseudogroup of $g$. Suppose $g \in \Gamma$, has the form $g=h_{m} \circ h_{m-1} \circ \cdots \circ h_{1}$ where each $h_{i} \in \Gamma_{0}$ and it is understood that each $h_{i}$ is restricted to some suitable part of its domain. Write 
$g_{p}=h_{p} \circ h_{p-1} \circ \cdots \circ h_{1}, 1 \leqslant p \leqslant m$. Thus, $g=g_{m}$. If $J \in g$ and if $J \subseteq$ $\operatorname{dom}(g)$, write $J_{p}=g_{p}(J), 1 \leqslant p \leqslant m$.

Definition. The element $g \in \Gamma$ is a simple chain based at $J$ if $J=\operatorname{dom}(g)$ and if $J, J_{1}, \ldots, J_{m}$ are distinct elements of $g$. If $J_{1}, \ldots, J_{m}$ are distinct and $J=J_{m}$, then $g$ is a simple loop based at $J$. Finally, if $h$ is a simple chain based at $J$ and $g$ is a simple loop based at $h(J)$, we call $h^{-1} g h$ a basic loop based at $J$.

Fix $[x, y]=J \in g$ and let $G$ be the set of $g \in \Gamma$ such that $J=\operatorname{dom}(g)$ and $g(J)=J$. Then $G$ is a group of orientation preserving $C^{2}$-diffeomorphisms of $J$ onto itself. Let $G_{0} \subseteq G$ denote the set of basic loops based at $J$. It is elementary that $G_{0}$ is a symmetric generating set for $G$. It is possible that $G_{0}$ is infinite. In the case in which $G_{0}$ is finite, the following is trivial, but we prove it in general.

(2.4) Theorem. Let $b \in(x, y)=\operatorname{int}(J)$. Then there is a point $a \in(x, b)$ such that $a<f(b)$, for all $f \in G_{0}$.

For the proof of (2.4), we need some elementary lemmas that will also be useful in (C). Fix $A>0$ and $B>0$ such that $h^{\prime}(t) \geqslant A$ and $\left|h^{\prime \prime}(t)\right|<B$, for each $h \in \Gamma_{0}$ and all $t \in \operatorname{dom}(h)$. The finiteness of $\Gamma_{0}$ and compactness of $\operatorname{dom}(h)$ make this possible. Set $\theta=B / A$.

(2.5) LEMMA. If $g=g_{m}=h_{m} \circ h_{m-1} \circ \cdots \circ h_{1}$, all $h_{i} \in \Gamma_{0}$, and $\operatorname{dom}(g)=J$ $\in \mathcal{G}$, and if $z, w \in J$, then

$$
g^{\prime}(z)<g^{\prime}(w) \exp \left(\theta \sum_{p=0}^{m-1}\left|J_{p}\right|\right)
$$

where $\left|J_{p}\right|$ denotes the length of $J_{p}$ and $J_{0}=J$.

Proof. Let $z_{0}=z, w_{0}=w, z_{p}=g_{p}(z), w_{p}=g_{p}(w), 1 \leqslant p \leqslant m-1$. By the chain rule

$$
\frac{g^{\prime}(z)}{g^{\prime}(w)}=\frac{h_{m}^{\prime}\left(z_{m-1}\right)}{h_{m}^{\prime}\left(w_{m-1}\right)} \cdot \frac{h_{m-1}^{\prime}\left(z_{m-2}\right)}{h_{m-1}^{\prime}\left(w_{m-2}\right)} \cdots \frac{h_{1}^{\prime}\left(z_{0}\right)}{h_{1}^{\prime}\left(w_{0}\right)} .
$$

Using the mean value theorem, write

$$
\begin{aligned}
\frac{h_{p+1}^{\prime}\left(z_{p}\right)}{h_{p+1}^{\prime}\left(w_{p}\right)} & =1+\frac{h_{p+1}^{\prime}\left(z_{p}\right)-h_{p+1}^{\prime}\left(w_{p}\right)}{h_{p+1}^{\prime}\left(w_{p}\right)} \\
& =1+\frac{h_{p+1}^{\prime \prime}\left(\xi_{p}\right)\left(z_{p}-w_{p}\right)}{h_{p+1}^{\prime}\left(w_{p}\right)}=1+\delta_{p}
\end{aligned}
$$

where $\xi_{p}$ lies between $z_{p}$ and $w_{p}, 0 \leqslant p<m-1$. Since $h_{p+1}$ is orientation preserving, $1+\delta_{p}>0$. Also, $\left|\delta_{p}\right| \leqslant \theta\left|z_{p}-w_{p}\right|$. Therefore

$$
\frac{g^{\prime}(z)}{g^{\prime}(w)}=\prod_{p=0}^{m-1}\left(1+\delta_{p}\right) \leqslant \prod_{p=0}^{m-1}\left(1+\left|\delta_{p}\right|\right) \leqslant \prod_{p=0}^{m-1} \exp \left(\left|\delta_{p}\right|\right)<\exp \left(\theta \sum_{p=0}^{m-1}\left|J_{p}\right|\right) \text {. }
$$

Let $c=3 \theta|q|$, where $|\mathcal{g}|$ denotes the total length of $\cup_{I \in \mathcal{G}} I \subset R$.

(2.6) LEMMA. If $g$ is a simple chain or a basic loop based at $J$ and if $z, w \in J$, then $g^{\prime}(z) \leqslant g^{\prime}(w) e^{c}$. 
Proof. By (2.5), $g^{\prime}(z) \leqslant g^{\prime}(w) \exp \left(\theta \sum_{p=0}^{m-1}\left|J_{p}\right|\right)$. Since an element $I \in g$ can appear at most three times in the list $J_{0}, J_{1}, \ldots, J_{m-1}$, it follows that $\sum_{p=0}^{m-1}\left|J_{p}\right|<$ $3|q|$.

(2.7) Corollary. If $g$ is a basic loop based at some $J \in \mathcal{G}$ and if $z \in J$, then $e^{-c} \leqslant g^{\prime}(z) \leqslant e^{c}$.

Proof. Since $g(J)=J$, the mean value theorem provides a point $w \in J$ such that $g^{\prime}(w)=1$. Also, one can interchange the roles of $z$ and $w$ in (2.6).

We can now give the proof of (2.4). Choose $a \in(x, b)$ so close to $x$ that $(b-x) /(a-x)>e^{c}$. Suppose there is some $f \in G_{0}$ such that $a \geqslant f(b)$. We must deduce a contradiction. Let $g=f^{-1} \in G_{0}$; hence assume $g(a)>b$. By the mean value theorem, there is $z$ between $x$ and $g(a)$ such that

$$
g^{\prime}(z)=(g(a)-g(x)) /(a-x)=(g(a)-x) /(a-x) .
$$

By (2.7), we obtain the contradiction

$$
e^{c} \geqslant g^{\prime}(z)=\frac{g(a)-x}{a-x} \geqslant \frac{b-x}{a-x}>e^{c} .
$$

(C) A generalized Kopell lemma. The lemma referred to is [K, Lemma la], long recognized to be significant in the theory of $C^{2}$-foliations. We continue to assume that $\Gamma$ is a finitely generated permuter pseudogroup of $q$. As in (B), we fix $[x, y]=J \in g$ and consider the group $G \subset \Gamma$.

Suppose $\mathscr{F}_{*} \subseteq \mathcal{G}, J_{*} \in \mathcal{F}_{*}$, and that a simple chain $h_{*} \in \Gamma$ has $J=\operatorname{dom}\left(h_{*}\right)$, $J_{*}=\operatorname{im}\left(h_{*}\right)$. With this data we define a subset $G_{*} \subset G$. Let $g \in \Gamma$ be a basic loop based at $J_{*}, g=h_{m} \circ h_{m-1} \circ \cdots \circ h_{1}$ as usual, and suppose that $g_{p}\left(J_{*}\right) \in g_{*}$, $0 \leqslant p \leqslant m$. Then $h_{*}^{-1} g h_{*} \in G$ and $G_{*}$ is to be the set of all such elements.

(2.8) THEOREM. If $x<a<b<y$, and if $\mathcal{g}_{*}$ as above has $\left|\mathcal{g}_{*}\right|$ sufficiently small, then $a<g(b)$, for each $g \in G_{*}$.

Before proving (2.8), we justify the claim that it generalizes the Kopell lemma. Let $R=[0,1]$ and let $f, g: R \rightarrow R$ be $C^{2}$ imbeddings with $f(0)=g(0)=0$. Suppose $f$ and $g$ commute and that $f$ is a contraction of $[0,1]$ to 0 . The Koppell lemma asserts that either $g$ fixes no point of $(0,1]$ or $g$ fixes all points. In order to obtain this as a consequence of $(2.8)$, let $z_{0} \in(0,1]$ be fixed by $g$, let $z_{k}=f^{k}\left(z_{0}\right), k \in \mathbf{Z}^{+}$, and remark that $g$ fixes each $z_{k}$. If $g=\left\{J_{k}=\left[z_{k+1}, z_{k}\right]: k \geqslant 0\right\}$, then $\Gamma_{0}=\{f, g\}$ generates a permuter pseudogroup of $g$. Remark that $g_{k}=g \mid J_{k}$ is a simple loop at $J_{k}$ and that $f^{q}: J_{k} \rightarrow J_{k+q}$ is a simple chain at $J_{k}$. Let $b \in\left(z_{1}, z_{0}\right)$ and suppose that $g_{0}(b) \neq b$. Without loss of generality, suppose $g_{0}(b)<b$ and take $a=g_{0}(b)$, $J=J_{0}$, in (2.8). Let $N \geqslant 0$ be an integer and let $g_{*}=\left\{J_{k}: k \geqslant N\right\}$. We can make $\left|g_{*}\right|$ as small as desired by taking $N$ large enough. Take $J_{*}=J_{N}, h_{*}=f^{N}: J_{0} \rightarrow$ $J_{N}$, and apply (2.8) to get

$$
a<h_{*}^{-1}\left(f^{-q} g_{N+q} f^{q}\right) h_{*}(b)=f^{-N-q} g_{N+q} f^{N+q}(b)
$$

for all $q \geqslant 0$. By commutativity, this reduces to the contradiction $a<g_{0}(b)=a$. This shows that $g_{0}$ fixes each point of $J_{0}$; hence also each point of $\left(0, z_{0}\right]=$ $\cup_{k>0} J_{k}$. Since $f^{r}(0,1] \subseteq\left(0, z_{0}\right]$, for $r$ sufficiently large, it follows that $g$ fixes each point of $(0,1]$. 
We turn to the proof of (2.8).

(2.9) Lemma. If $r<1<s$, then, for $\left|\mathcal{F}_{*}\right|$ sufficiently small, and for all $g \in$ $h_{*} \circ G_{*} \circ h_{*}^{-1}$, it is true that $r<g^{\prime}<s$ uniformly on $J_{*}$.

Proof. Set $c_{*}=3 \theta\left|g_{*}\right|$. Then, for $\left|g_{*}\right|$ sufficiently small, we have $e^{c_{*}}<$ $\min \{s, 1 / r\}$. But, applying (2.5) as in the proof of (2.7), we also obtain $e^{-c_{*}}<g^{\prime}<$ $e^{c_{*}}$, uniformly on $J_{*}$, for each $g \in h_{*} \circ G_{*} \circ h_{*}^{-1}$.

Choose $s>1$ such that $s-1<e^{-c}(b-a) /(a-x)$. For $g_{*}$ as in (2.9), $g^{\prime}(t)<$ $s$, for all $t \in J_{*}$ and for all $g \in h_{*} \circ G_{*} \circ h_{*}^{-1}$. Suppose there is $f \in G_{*}$ with $a \geqslant f(b)$. Set $g=h_{*} \circ f^{-1} \circ h_{*}^{-1}, a_{*}=h_{*}(a), b_{*}=h_{*}(b)$, so that $g\left(a_{*}\right) \geqslant b_{*}$. We will show that there is a point $t \in J_{*}$ such that $g^{\prime}(t)>s$, a contradiction.

Indeed, set $x_{*}=h_{*}(x)$ and obtain

$$
\begin{aligned}
\left(g\left(a_{*}\right)-a_{*}\right) /\left(a_{*}-x_{*}\right) & \geqslant\left(b_{*}-a_{*}\right) /\left(a_{*}-x_{*}\right) \\
& =h_{*}^{\prime}(\xi)(b-a) / h_{*}^{\prime}(\zeta)(a-x) \\
& \geqslant e^{-c}(b-a) /(a-x)>s-1 .
\end{aligned}
$$

Here, the mean value theorem provides $\xi \in(a, b) \subset J$, and $\zeta \in(x, a) \subset J$, and the second last inequality is given by (2.6). Thus, $\left(g\left(a_{*}\right)-x_{*}\right) /\left(a_{*}-x_{*}\right)>s$, so the mean value theorem provides $t \in\left(x_{*}, a_{*}\right) \subset J_{*}$ such that $g^{\prime}(t)>s$.

Remark that we did not use the full strength of (2.9). But the fact that $g^{\prime}$ is uniformly as close to 1 as desired, $\forall g \in h_{*} \circ G_{*} \circ h_{*}^{-1}$, as $\left|g_{*}\right| \rightarrow 0$, seems interesting and potentially useful.

3. The structure of open, saturated sets. In this section we sketch and adapt to our purposes some important results and viewpoints due to G. Hector (thesis) and $P$. Dippolito [Di], culminating in a proof of (3.0).

Let $\left\{\left(W_{i}, \varphi_{i}\right)\right\}_{i=1}^{m}$ be a biregular cover of $M$. For suitable small $\varepsilon, \delta>0$ we can assume that the interiors of the sets $W_{i}(\delta, \varepsilon)=\varphi_{i}\left(D^{n-1} \times[\delta-1,1-\varepsilon]\right)$ also cover $M$.

Let $U \subset M$ be an open, $\mathscr{F}$-saturated set. Renumber the sets $\left\{W_{i}\right\}$ so that $W_{i} \subset U$ if and only if $q<i \leqslant m$, some $q \leqslant m$. Thus, if $1 \leqslant i \leqslant q$, the connected components of $W_{i} \cap U$ are sets of one of the following three kinds:

$$
\begin{aligned}
W_{i}^{+} & =\varphi_{i}\left(D^{n-1} \times\left(a_{i}, 1\right]\right), \\
W_{i}^{-} & =\varphi_{i}\left(D^{n-1} \times\left[-1, b_{i}\right)\right), \\
W_{i, k} & =\varphi_{i}\left(D^{n-1} \times\left(a_{i, k}, b_{i, k}\right)\right)
\end{aligned}
$$

where $k$ may run over a finite or infinite indexing set. We define $\delta U$ to be the union of all plaques of any of the following forms:

$$
\begin{aligned}
& P_{i}^{+}=\varphi_{i}\left(D^{n-1} \times\left\{a_{i}\right\}\right), \quad P_{i}^{-}=\varphi_{i}\left(D^{n-1} \times\left\{b_{i}\right\}\right), \\
& P_{i, k}^{+}=\varphi_{i}\left(D^{n-1} \times\left\{a_{i, k}\right\}\right), \quad P_{i, k}^{-}=\varphi_{i}\left(D^{n-1} \times\left\{b_{i, k}\right\}\right),
\end{aligned}
$$

where $1 \leqslant i \leqslant q$. Thus, $\delta U$ is the union of the "boundary leaves" of $U$, but it need not be the set theoretic boundary of $U$. 
It is useful to form a manifold $\hat{U}$ with boundary by cutting the manifold $M$ apart along the leaves of $\delta U$ and keeping only the components meeting $U$. More formally, let $\iota: U \rightarrow M$ be the imbedding, pull back a Riemannian metric from $M$ to $U$ via $\iota$, and let $\hat{U}$ be the completion of $U$ in this metric. Let $\hat{\imath}: \hat{U} \rightarrow M$ be the canonical extension of $\iota$ to an immersion such that $\hat{\imath}(\partial \hat{U})=\delta U$. By the transverse orientability of $\mathcal{F}, \hat{\imath}$ maps each component of $\partial \hat{U}$ diffeomorphically onto a leaf of $\delta U$, but two distinct boundary components may be identified.

One obtains foliations $\hat{\mathscr{F}}$ and $\hat{\mathcal{L}}$ on $\hat{U}$ by pulling back $\mathscr{F}$ and $\mathcal{L}$ via $\hat{\imath}$. A locally finite atlas of $(\hat{\mathscr{F}}, \hat{\mathfrak{e}})$ coordinate charts on $\hat{U}$ is defined by adjoining to $\left\{W_{i}\right\}_{q<i<m}$ all of the sets

$$
\begin{aligned}
\hat{W}_{i}^{+} & =\varphi_{i}\left(D^{n-1} \times\left[a_{i}, 1\right]\right)=W_{i}^{+} \cup P_{i}^{+}, \\
\hat{W}_{i}^{-} & =\varphi_{i}\left(D^{n-1} \times\left[-1, b_{i}\right]\right)=W_{i}^{-} \cup P_{i}^{-}, \\
\hat{W}_{i, k} & =\varphi_{i}\left(D^{n-1} \times\left[a_{i, k}, b_{i, k}\right]\right)=W_{i, k} \cup P_{i, k}^{+} \cup P_{i, k}^{-},
\end{aligned}
$$

$1 \leqslant i \leqslant q$, imbedding these in $\hat{U}$ by $\hat{\imath}^{-1}$, and suitably reparametrizing the various (well-defined) restrictions of $\hat{\imath}^{-1} \circ \varphi_{i}$.

We will suppress further reference to $\hat{\imath}$ and $\hat{\imath}^{-1}$.

The following is a trivial observation.

(3.1) LEMMA. If $U$ is connected and if each leaf of $\hat{\mathfrak{L}}$ is a compact arc, then $\hat{U}$ can be identified (via a suitable diffeomorphism) with $L^{+} \times I$ where $L^{+}$is a leaf of $\hat{\mathcal{F}}$ and a component of $\partial \hat{U}$ and each $\{x\} \times I$ is a leaf of $\hat{\mathfrak{L}}$. In this case, $\partial \hat{U}$ has two mutually diffeomorphic components, namely $L^{+}=$the union of all $P_{i}^{+}$and $P_{i, k}^{+}$and $L^{-}=$the union of all $P_{i}^{-}$and $P_{i, k}^{-}$.

DEFINITION. In the situation of (3.1), $\hat{U}$ will be called a foliated product.

The following is a very useful property of foliated products. Verification is straightforward and will be left to the reader.

(3.2) LEMMA. If $\hat{U}$ is a foliated product, then every element of the holonomy pseudogroup of $\mathcal{F}$ defined by a path along some leaf of $U \cup \delta U$ extends to an element defined simultaneously by paths along every leaf of $U \cup \delta U$. Furthermore, if $L_{1}$ and $L_{2}$ are the leaves (possibly identical) of $\delta U$ and if $L_{0} \subset U$ is a leaf of $\mathscr{F}$, then $\lim \left(L_{1}\right)=\lim \left(L_{2}\right) \subseteq \lim \left(L_{0}\right)$.

Except for a compact subset, every $\hat{U}$ looks like a bunch of foliated products. This statement is made completely precise in [Di, Theorem 1], but here we will be content with the following.

(3.3) Proposition. Let $L$ be a component of $\partial \hat{U}$. Then there is a compact, connected, $(n-1)$-dimensional submanifold $A \subset L$ such that the components $B^{1}, \ldots, B^{r}$ of $L-\operatorname{int}(A)$ are each unbounded, $B^{j} \cap A$ is a component of $\partial A$, $1 \leqslant j \leqslant r$, and the $\hat{\mathfrak{L}}$-saturation of $B^{j}$ is (diffeomorphic to) $B^{j} \times I$ where each $\{x\} \times I$ is a leaf of $\hat{\mathfrak{E}}, 1 \leqslant j \leqslant r$. Furthermore, at most finitely many components of $\hat{U}$ fail to be foliated products. 
Proof. If $L$ is compact, choose $A=L$, so that the assertions hold vacuously. If $U$ lies on the positive side of $L$, choose $A$ so that it contains each plaque in $L$ of the form $P_{i}{ }^{+}$. If $U$ lies on the negative side of $L$, then one chooses $A$ to cover each plaque in $L$ of the form $P_{i}^{-}$. There is no problem making sure that $A$ is connected, that each $B^{j} \cap A$ is connected, and that $L-A$ has no bounded component. Furthermore, there can only be finitely many components $L$ of $\partial \hat{U}$ containing a plaque of the form $P_{i}^{+}$, and, if $L$ is not one of these, we take $A=\varnothing$. Then each $B^{j}$ is only met by plaques of the form $P_{i, k}^{+}$(respectively, $P_{i, k}^{-}$). The conclusions are immediate.

We consider a leaf $L$ of $\mathscr{F}$ with a proper side. For definiteness, assume that the positive side of $L$ is proper and fix a compact subarc $J=[x, y]$ of a leaf of $\mathcal{L}$ such that $J \cap L=\{x\}$. Let $J^{+}=(x, y]$. Let $\Gamma$ be the pseudogroup on $J^{+}$defined by the holonomy along $L$.

Definition. The pseudogroup $\Gamma$ is unbounded if, for each $t \in J^{+}, \Gamma(t)$ clusters at $x$. Equivalently, for each $t \in J^{+}$there is $g \in \Gamma$ such that $g(t) \neq t[\mathbf{P} 1$, Lemma 5.1].

A result of Sacksteder and Schwartz [S-S, Theorem 1], or at least its proof, shows that, if some leaf $L^{\prime}$ of $\mathscr{F}$ approaches $L$ from the positive side, then $J$ can be chosen so that $\Gamma$ is unbounded. A recent result of Dippolito [Di, Theorem 3] sharpens this considerably.

(3.4) Theorem (Dippolito). If, for every choice of $J, \Gamma$ is not unbounded, then for each fixed point $t$ of $\Gamma$ sufficiently near $x$, there is an imbedding $\Psi_{t}: L \times[0,1] \rightarrow M$ such that

(1) $\Psi_{t}(z, 0)=z, \forall z \in L$

(2) $\Psi_{t}(\{z\} \times[0,1])$ is contained in a leaf of $\mathcal{L}, \forall z \in L$;

(3) $\Psi_{t}(L \times\{1\})$ is the leaf of $\mathscr{F}$ through $t$.

Proof. Let $U$ be the $\mathscr{F}$-saturation of the interior of $J$. Then $L \subset \partial \hat{U}$ and we let $A, B^{1}, \ldots, B^{r}$ be as in (3.3). We can assume that $J \cap L$ is a point of $A$. Let $L_{t} \subset U$ be the leaf through the $\Gamma$-fixed point $t$. If $t$ is sufficiently near 0 , then the fact that $A$ is connected and that $\pi_{1}(A)$ is finitely generated implies, in a standard way, that a subset of $L_{t}$ projects along the leaves of $\hat{\mathfrak{L}}$ diffeomorphically onto $A$. The inverse of this projection is an imbedding $p: A \rightarrow L_{t}$. Each $B^{j} \cap A$ is connected and each path in $B^{j}$ starting on $B^{j} \cap A$ lifts uniquely to $L_{t}$ with initial point on $p\left(B^{j} \cap A\right)$ (by the structure of foliated products), loops lifting to loops (since $t$ is $\Gamma$-fixed). Thus, $p$ extends to an open imbedding $p: L \rightarrow L_{t}$. It is elementary to check that $p(L)$ is also a closed subset of $L_{t}$, so $p$ maps $L$ diffeomorphically onto $L_{t}$. The desired conclusion follows easily.

The next two lemmas correspond to Proposition 1 and Proposition 2 respectively in [Di].

(3.5) Lemma. Let $U_{1} \supseteq U_{2} \supseteq \cdots \supseteq U_{j} \supseteq \cdots$ be a nest of open, connected, $\mathscr{F}$-saturated sets such that $\cap U_{j}$ has empty interior. Then, for $j$ sufficiently large, $\hat{U}_{j}$ is a foliated product. 
Proof. Let $X=\cap U_{j}$. For suitable small $\varepsilon, \delta>0$, the modified $(\mathscr{F}, \mathcal{E})$ atlas with images $W_{i}(\delta, \varepsilon), \quad 1 \leqslant i \leqslant m$, is such that no plaque of the form $\varphi_{i}\left(D^{n-1} \times\{\delta-1\}\right)$ or $\varphi_{i}\left(D^{n-1} \times\{1-\varepsilon\}\right)$ meets $X$. Thus, without loss of generality, we assume that no $\varphi_{i}\left(D^{n-1} \times\{ \pm 1\}\right)$ meets $X$. It follows that, for suitably large $j$, none of these plaques meets $U_{j}$. The desired conclusion is immediate.

(3.6) LEMMA. If $U$ is an open, connected, $\mathcal{F}$-saturated set, then $\partial \hat{U}$ has only a finite number of components.

Proof. Let $L$ be a component of $\partial \hat{U}$. If $\hat{U}$ is a foliated product, we are done. Otherwise, $L$ contains at least one plaque of the form $P_{i}^{+}$or $P_{i}^{-}$. Since there are only finitely many such plaques, $\partial \hat{U}$ can contain only finitely many components $L$.

For the proof of (3.0), we will need the following result.

(3.7) Lemma. Let $U \subseteq M$ be an open, connected, nonempty $\mathscr{F}$-saturated set and let $L$ be a leaf of $\mathscr{F} \mid U$. Then there is a finite system $K_{1}, \ldots, K_{p}$ of compact, disjoint subarcs of leaves of $\mathcal{L}$, each $K_{i} \subset U$, such that every leaf of $\bar{L} \cap U$ meets the interior of at least one $K_{i}$.

Before proving (3.7), we will show how it implies (3.0). Let $K=\cup_{i=1}^{p} K_{i}$ and let $\Gamma$ be the holonomy pseudogroup on $K$ defined by $\mathscr{F}$. Then $\bar{L} \cap K$ is a compact, nonempty, $\Gamma$-invariant set and, by Zorn's lemma, there exists a minimal, nonempty, compact, $\Gamma$-invariant subset $C \subseteq \bar{L} \cap K$. Let $X$ denote the $\mathscr{F}$-saturation of $C$. We claim that $X$ is closed in $U$. Indeed, $X \subseteq \bar{L} \cap U$ and, if $L_{0}$ is a leaf of $\mathscr{F} \mid U$ approached by a sequence of points of $X$, then, since $L_{0}$ meets some arc $K_{i}$ in a point $x_{0}$ interior to $K_{i}, x_{0}$ is approached in $K_{i}$ by a sequence of points of $C$. It follows that $x_{0} \in C$; hence that $L_{0} \subseteq X$. Since $C$ is minimal, it also follows that $X$ is a minimal set of $\mathscr{F} \mid U$.

We commence the proof of (3.7). The assertion is evident for the case in which $U=M$, so we assume $\delta U \neq \varnothing$. Let $L_{1}, \ldots, L_{q}$ denote the leaves of $\partial \hat{U}$, finite in number by (3.6). Let $L_{i}=A_{i} \cup B_{i}^{1} \cup \cdots \cup B_{i}^{r(i)}$ denote the decomposition obtained in (3.3), $1 \leqslant i \leqslant q$. It is possible that $r(i)=0$, which is to say that $L_{i}=A_{i}$, a compact leaf.

Each minimal set of $\mathscr{F}$ in $\bar{L}$ either lies entirely in $U$ or entirely in $M-U$. Since $\varnothing \neq U \neq M$, either each such minimal set is exceptional or it is a compact leaf. By [La], there are at most finitely many exceptional minimal sets of $\mathscr{F}$ in $M$ and it is well known that $\bar{L}$ can only contain finitely many compact leaves (use [Ha, 3.2]). Thus, there are at most finitely many minimal sets $X_{1}, \ldots, X_{s}$ of $\mathscr{F}$ contained in $\bar{L} \cap U, s \geqslant 0$. If $s>0$, we choose $K_{1}, \ldots, K_{s} \subset U$, compact subarcs of leaves of $\mathcal{L}$ such that each leaf of $X_{j}$ meets $\operatorname{int}\left(K_{j}\right), 1<j<s$.

Claim. Every leaf of $\bar{L} \cap U \subset \hat{U}$ either meets the interior of some $K_{j}, 1<j<s$, or approaches in $\hat{U}$ a compact leaf $L_{i} \subseteq \partial \hat{U}$, or enters the $\hat{\mathcal{L}}$-saturation of some $B_{i}^{j}$.

Proof. Let $L^{\prime}$ be a leaf of $\bar{L} \cap U$ and let $X \subseteq \bar{L}$ be a minimal set approached by $L^{\prime}$. If $X \subset U$, then $L^{\prime}$ meets the interior of some $K_{i}, 1 \leqslant i<s$. Suppose $X \subseteq M-U$. If $X$ contains a leaf of $\delta U$ which, as a leaf of $\partial \hat{U}$, is approached by $L^{\prime}$ 
in $\hat{U}$, we are done. Otherwise, take a leaf $J$ of $\mathcal{L}$ meeting $X$ and a point $x \in X \cap J$. There is a sequence $\left\{x_{k}\right\}_{k \geqslant 1} \subseteq L^{\prime} \cap J$ converging to $x$, say from the right. Thus $\left[x, x_{1}\right]$ meets $\delta U$ infinitely often and there are arcs $\left[c_{k}, d_{k}\right] \subset J$, each a leaf of $\hat{\mathcal{L}}$, with $x_{k} \in\left(c_{k}, d_{k}\right)$. For $k$ sufficiently large, $\left[c_{k}, d_{k}\right]=[c, d]$ must be a leaf of $\hat{\mathcal{L}}$ in the $\hat{\mathcal{L}}$-saturation of some $B_{i}^{j}$.

Select a finite system $I_{s+1}, \ldots, I_{p} \subset \hat{U}$ of compact, disjoint subarcs of $\hat{\mathfrak{L}}$ such that, for each $B_{i}^{j}$, a leaf of $\hat{\mathcal{L}}$ in the $\hat{\mathfrak{E}}$-saturation of $B_{i}^{j}$ occurs in the list, and such that, if $L_{i} \subseteq \partial \hat{U}$ is a compact leaf, then some $I_{k}$ has one end on $L_{i}$ and the other end in $U$. If we can find a compact subinterval $K_{k} \subset I_{k} \cap U, s+1 \leqslant k \leqslant p$, such that every leaf of $\bar{L}$ meeting $I_{k} \cap U$ actually meets the interior of $K_{k}$, then (3.7) will follow from the above claim.

Let $I_{k}=[c, d]$, and suppose $c \in L_{i} \subseteq \partial \hat{U}$ (if $d \in L_{i}$, we argue in a parallel way). If the holonomy subpseudogroup $\Gamma_{i}$ on $[c, d]$, induced by loops on $L_{i}$ based at $c$, is not unbounded near $c$, then (3.4) guarantees the existence of $a \in(c, d)$ such that $\bar{L} \cap(c, a]=\varnothing$. In this case, we can take $a$ as the lower endpoint of $K_{k}$. The following, therefore, will be enough to establish the existence of $K_{k}$; hence it will complete the proof of (3.7).

(3.8) Lemma. Let $L_{i},[c, d]$, and $\Gamma_{i}$ be as above. If $\Gamma_{i}$ is unbounded near $c$, then there is $[a, b] \subset(c, d)$ such that every leaf of $\mathscr{F}$ that meets $(c, b]$ also meets $(a, b)$.

For the proof of (3.8), we need the following. It is here that differentiability of class $C^{2}$ is used.

(3.9) Lemma. There is $[a, b] \subset(c, d)$, as near to $c$ as desired, and a generating set $\Gamma_{*} \subset \Gamma_{i}\left[[c, b]\right.$, such that, for each $f \in \Gamma_{*},[c, b]=\operatorname{dom}(f)$ and $a<f(b)<b$.

Proof of (3.8) UsING (3.9). Choose $b$ near enough to $c$ that $\Gamma_{i}$ is unbounded on $(c, b]$. Thus, there is some $f \in \Gamma_{*}$ such that $a<f(b)<b$, so the only way (3.8) can fail is that there exists $z \in(c, a]$ such that $z_{0}=\sup \left(\Gamma_{i}(z) \cap(c, b]\right)<a$. For each $g \in \Gamma_{*}, g\left(z_{0}\right)<g(b) \leqslant b$ and so $g\left(z_{0}\right) \leqslant z_{0}$. By unboundedness, some $g \in \Gamma_{*}$ satisfies $g\left(z_{0}\right)<z_{0}$. But $z_{0} \leqslant a<g(b)$, so $g\left(z_{0}\right)<z_{0}<g(b)$; hence $g^{-1}$ is defined at $z_{0}$ and $z_{0}<g^{-1}\left(z_{0}\right)<b$. This contradicts the definition of $z_{0}$.

Proof of (3.9). We need only consider the case in which $L_{i}$ is noncompact. Otherwise, $\pi_{1}\left(L_{i}\right)$ is finitely generated and one easily obtains a finite set $\Gamma_{*}$ as in (3.9). Thus, the decomposition $L_{i}=A_{i} \cup B_{i}^{1} \cup \cdots \cup B_{i}^{r(i)}$ has $r(i)>1$. Fix a biregular cover $\left\{\left(\varphi_{k}, W_{k}\right)\right\}_{k=1}^{m}$. By suitably enlarging $A_{i}$, if necessary, arrange that, for each $k, W_{k}$ will meet the interior of a leaf $J$ of $\hat{\mathfrak{L}}$ in the $\hat{\mathcal{L}}$-saturation of some $B_{i}^{j}$ if and only if $J \subset W_{k}$. Fix $B=B_{i}^{j}$ and in each $W_{k}$ choose $R_{k}=\varphi_{k}(\{x\} \times I)$, arrange that these arcs be disjoint, and let $R$ denote their union. Let $g$ denote the set of intervals $J \subset R$ where $J$ is a leaf of $\hat{\mathfrak{L}}$ in the $\hat{\mathcal{L}}$-saturation of $B$. If $W_{k} \cap W_{h} \neq \varnothing$, there is a holonomy transition function $g_{k h}$ with domain a compact subinterval of $R_{k}$ and image a compact subinterval of $R_{h}$.

Consider those intervals $J \in \mathcal{G}$ lying in $\operatorname{dom}\left(g_{k h}\right)$ such that $g_{k h}(J) \notin g$. There are at most finitely many such intervals $J_{1}, \ldots, J_{r}$ because, by the compactness of $\partial B$, there are at most finitely many plaques in $W_{k} \cup W_{h}$ that meet $\partial B$. The 
components of $\operatorname{dom}\left(g_{k h}\right)-\cup_{i=1}^{r} \operatorname{int}\left(J_{i}\right)$ are compact intervals, finite in number. The restrictions of $g_{k h}$ to these intervals are permuters of $\mathcal{g}$. Since there are only finitely many $g_{k h}$, we proceed as above with all of them and obtain a finite set of permuters of $g$. Let $\tilde{\Gamma}$ denote the pseudogroup (of class $C^{2}$ ) on $R$ generated by these permuters.

The above paragraph has set the stage for an application of (2.4). Fix $J=[x, y]$ $\in \mathcal{G}$ and, for definiteness, consider the case $x \in B$. Let $G$ be the group of $C^{2}$-diffeomorphisms of $J$ onto itself defined (as in $\$ 2(B)$ ) by $\tilde{\Gamma}$, and observe that $G$ contains every holonomy transformation of $J$ onto itself produced by loops on $B$ based at $x$. Let $G_{0}$ be the symmetric generating set of $G$ as in (2.4). Given $b^{\prime} \in(x, y)$, we can use (2.4) to find $a^{\prime} \in\left(x, b^{\prime}\right)$ as close to $x$ as desired, satisfying $a^{\prime}<f\left(b^{\prime}\right)$ for all $f \in G_{0}$.

By Van Kampen's theorem, $\pi_{1}\left(L_{i}, c\right)$ is generated by the image of $\pi_{1}\left(A_{i}\right) \cup$ $\pi_{1}\left(B_{i}^{1}\right) \cup \cdots \cup \pi_{1}\left(B_{i}^{r(i)}\right)$ suitably mapped into $\pi_{1}\left(L_{i}, c\right)$. Arguing as above for each $B_{i}^{j}$ and using the fact that $\pi_{1}\left(A_{i}\right)$ is finitely generated, we readily choose $[a, b] \subset$ $(c, d)$ and $\Gamma_{*}$ as specified in (3.9).

The proof of (3.0) is complete.

4. The structure of the set of local minimal sets. Let $C_{k} \subseteq M_{k}, k \geqslant 0$, be the sets defined as in the Statements of Results. It will be convenient to set $C_{-1}=M_{-1}=\varnothing$. Our main aim in this section is to prove (4.0).

We emphasize the convention of $\$ 1(\mathrm{E})$.

In the following two lemmas, $L$ denotes an arbitrary leaf of $\mathscr{F}$.

(4.1) LEMMA. Let $Y \subseteq \bar{L}$ be a compact, $\mathcal{F}$-saturated subset. Let $U$ be the component of $M-Y$ containing $L$, and let $L_{1}, \ldots, L_{r}$ be the leaves of $\delta U$. Then $Y=\bar{L}_{1} \cup \cdots \cup \bar{L}_{r}$.

Proof. If $Y=\varnothing$, then $\delta U=\varnothing$ and there is nothing to prove. The fact that $\delta U$ is a finite union of leaves is from (3.6). Evidently $\bar{L}_{1} \cup \cdots \cup \bar{L}_{r} \subseteq Y$. Let $L_{0} \subseteq Y$ be a leaf of $\mathscr{F}$. Since $L$ is asymptotic to $L_{0}$, a transverse arc to $L_{0}$ is met by $L$ in a set of points that contains a sequence $\left\{x_{m}\right\}_{m>1}$ converging to $x_{0} \in L_{0}$. Either $x_{0} \in$ $\delta U$, or the arc from $x_{m}$ to $x_{0}$ must cross leaves of $\delta U$. Since there are only finitely many such leaves, it follows that $L_{0} \subseteq \bar{L}_{i}$, some $i$. Thus, $Y \subseteq \bar{L}_{1} \cup \cdots \cup \bar{L}_{r}$ and equality holds.

(4.2) LEMma. If $X_{1} \subset X_{2} \subset \cdots \subset X_{i} \subset \cdots \subset \bar{L}$ is an infinite nest of compact $\mathcal{F}$-saturated subsets, then $X_{*}=\cup_{i=1}^{\infty} X_{i}$ is dense in $\bar{L}$.

Proof. If $\bar{X}_{*} \neq \bar{L}$, let $U$ be the component of $M-\bar{X}_{*}$ containing $L$. Let $L_{1}, \ldots, L_{r}$ be the leaves of $\delta U$. By (4.1) $\bar{X}_{*}=\bar{L}_{1} \cup \cdots \cup \bar{L}_{r}$. We know that some $L_{i} \in X_{*}$, since otherwise there would be a sufficiently large integer $k$ such that $\bar{X}_{*}=\bar{L}_{1} \cup \cdots \cup \bar{L}_{r} \subseteq X_{k}$, a contradiction. Assume $L_{1} \_X_{*}$. If some $L_{i}$ is asymptotic to $L_{1}$, then $L_{i} \notin X_{*}$. Thus, without loss of generality, we assume that $\bar{L}_{1}$ is not properly contained in any $\bar{L}_{i}$. Let $y \in L_{1}$ and let $J$ be a compact subarc of a leaf of $\mathcal{L}$ such that $y \in \operatorname{int}(J)$. Let $x_{k} \in X_{k} \cap J$ such that $\lim _{k \rightarrow \infty}\left(x_{k}\right)=y$. Without loss of generality, assume that $x_{k} \notin \delta U$, for all $k$. Since $L \cap J$ clusters at 
$x_{k}$, for each $k$, so does $\delta U \cap J$. It follows that some leaf $L_{i}$ is asymptotic to $L_{1}$. By the above, if $i \neq 1, L_{1}$ is also asymptotic to $L_{i}$ and, in any case, $L_{1}$ is asymptotic to itself. Since $L_{1}$ is a leaf of $\delta U$, it has a proper side. Thus, (2.3) and the remark following that proposition imply that, for some $k$, the leaf through $x_{k}$ is asymptotic to the leaf $L_{1}$ through $y$. That is, $L_{1} \subseteq X_{k} \subset X_{*}$, a contradiction. Consequently, $\bar{X}_{*}=\bar{L}$ as desired.

We can now establish the first assertion of (4.0). We abbreviate " local minimal set" by LMS.

(4.3) ThEOREM. Each LMS of $\mathscr{F}$ is at some finite level.

Proof. Let $X$ be a LMS. Let $L$ be a leaf in $X$. Choose a minimal set $X_{1} \subseteq \bar{X}=\bar{L}$. If $X_{1}=\bar{L}$, then $X=X_{1}$ is at level 0 . If $X_{1} \subset \bar{L}$, let $U$ denote the component of $M-X_{1}$ containing $L$. Then $X \subseteq U$. If $\bar{L} \cap U=X$, then $X$ is at level 1. Otherwise, by (3.0), there is a minimal set $Y \subset \bar{L} \cap U$ of $\mathscr{F} \mid U$, and $X_{2}=X_{1} \cup Y$ is compact. Necessarily, $Y$ is a LMS at level at most 1 . Proceeding in this way, we produce a nest $X_{1} \subset X_{2} \subset \cdots \subset X_{k} \subseteq \bar{L}$ of compact, $\mathscr{F}$-saturated sets such that $X_{i}-X_{i-1}$ is a LMS at finite level, $2 \leqslant i \leqslant k$. If $X_{k}=\bar{L}$, then $X=X_{k}-X_{k-1}$ and we are done. If no such integer $k$ is ever reached, we obtain an infinite nest $X_{1} \subset X_{2} \subset \cdots \subset \bar{L}$. By (4.2), $X_{*}=\cup_{i=1}^{\infty} X_{i}$ is dense in $\bar{L}$. But $X_{*} \subseteq \bar{L}-X$ and, by the definition of LMS, $\bar{L}-X$ is compact. This is a contradiction.

We will prove the remaining assertions of (4.0) by induction on $k$. It will be convenient to commence the induction with the vacuously true case $k=-1$.

Suppose that $M_{k}$ (respectively, $C_{k}$ ) is compact, some $k \geqslant-1$. Let $U=M-M_{k}$ (respectively, $U=M-C_{k}$ ), an open, $\mathscr{F}$-saturated set, generally not connected. Let $\left\{Y_{i}\right\}_{i=1}^{\infty}$ be a sequence of distinct LMS at level $k+1$ (respectively, distinct, totally proper leaves at level $k+1$ ). Then $Y_{i} \subseteq U$ and $\bar{Y}_{i}-Y_{i} \subseteq M_{k}$ (respectively, $\left.C_{k}\right), \forall i \geqslant 1$. Suppose that $x_{i} \in Y_{i}$ and that $\lim _{i \rightarrow \infty}\left(x_{i}\right)=x \in M$. Let $L$ be the leaf of $\mathscr{F}$ through $x$. We must show $L \subseteq M_{k+1}$ (respectively, $L \subseteq C_{k+1}$ ).

If $L \subseteq M_{k}$ (respectively, $L \subseteq C_{k}$ ), we are done. Thus, we assume $L \subset U$. Let $U_{0}$ denote the component of $U$ containing $L$. By (3.0), let $Y \subseteq \bar{L} \cap U_{0}$ be a minimal set of $\mathscr{F} \mid U_{0}$.

(4.4) Lemma. The set $Y$ reduces to a single proper leaf.

Proof. Let $y \in Y$. Without loss of generality, assume that a transverse arc $[y, z) \subset U_{0}$ and points $y_{i} \in Y_{i}$ are such that $\left\{y_{i}\right\}_{i=1}^{\infty} \subset[y, z)$ and $\lim _{i \rightarrow \infty}\left(y_{i}\right)=y$. If $Y=U_{0}$, then every $Y_{i}=U_{0}$, contradicting the assumption that these LMS are distinct. If $Y$ is of exceptional type, (2.2) and the remark following the proof of (2.3) allow us to have selected $y$ so that the leaf through $y$ has an element of 2-sided contracting holonomy. Again, for $i$ large, this leads to the contradiction $Y_{i}=Y_{i+1}$ $=\cdots=Y$. It remains that $Y$ reduces to a proper leaf.

(4.5) Proposition. The set $Y$ coincides with the leaf $L$, hence $L \subseteq M_{k+1}$ (respectively, $L \subseteq C_{k+1}$ ). 
Proof. Otherwise, $L$ is asymptotic to the leaf $L^{\prime}=Y$, so we let $y \in L^{\prime}$ and $[y, z$ ) be such that $L \cap[y, z)$ clusters at $y$. Since each point of $L \cap[y, z)$ is the limit of a sequence of points $\left\{z_{i} \in Y_{i} \cap[y, z)\right\}_{i=1}^{\infty}$, and since we can assume that each $Y_{i} \neq Y$, we obtain a sequence $\left\{y_{i_{j}} \in Y_{i_{j}} \cap[y, z)\right\}_{j=1}^{\infty}$ of closest points to $y, y=$ $\lim _{j \rightarrow \infty}\left(y_{i}\right)$. Let $L_{j}$ be the leaf through $y_{i,}$. By (3.4), there is an imbedding $\varphi_{j}: L^{\prime} \times$ $[0,1] \rightarrow M$, all large values of $j$, such that $\varphi_{j}\left(L^{\prime} \times\{0\}\right)=L^{\prime}$ and $\varphi_{j}\left(L^{\prime} \times\{1\}\right)=$ $L_{j}$, and each $\varphi_{j}(\{w\} \times[0,1]), w \in L^{\prime}$, is a subarc of a leaf of $\mathcal{L}$. But $L$ is a leaf such that $L \cap[y, z)$ clusters at $y$; hence it would have to intersect leaves $L_{j}$, a contradiction.

This completes the inductive proof of the following.

(4.6) TheOREM. For each $k \geqslant 0, M_{k}$ and $C_{k}$ are compact sets.

(4.7) THEOREM. If $k \geqslant 0$ and if $L$ is a leaf of $\mathscr{F}$, then $\bar{L} \cap M_{k}$ is a finite union of LMS.

Proof. If a LMS meets $\bar{L}$, it is contained in $\bar{L}$, so $\bar{L} \cap M_{k}$ is a union of LMS.

Let the inductive hypothesis be that, for any leaf $L$ of $\mathscr{F}, \bar{L} \cap M_{k-1}$ is a finite union of LMS, $k \geqslant 0$. Then this holds vacuously for $k=0$.

Assume the hypothesis for all $k<j$. Let $L$ be a leaf of $\mathscr{F}$ and consider $\bar{L} \cap M_{j}$. If $\bar{L} \cap M_{j}=\bar{L}$, then $L \subset M_{j}$ and $Y=\bar{L}-\bar{L} \cap M_{j-1}$ is a LMS at level $j$. Thus, by the inductive hypothesis, $\bar{L} \cap M_{j}=\bar{L}=Y \cup\left(\bar{L} \cap M_{j-1}\right)$ is a finite union of LMS.

If $\bar{L} \cap M_{j} \neq \bar{L}$, let $U$ be the component of $\underline{M}-\left(\bar{L} \cap M_{j}\right)$ containing $L$. Let $L_{1}, \ldots, L_{r}$ be the leaves of $\delta U$ and remark that $\bar{L}_{i} \cap M_{j}=\bar{L}_{i}, 1<i<r$; hence $\bar{L}_{i}$ is a finite union of LMS as above. By (4.1), $\bar{L} \cap M_{j}=\bar{L}_{1} \cup \cdots \cup \bar{L}_{r}$.

It is an immediate corollary of (4.7) that $\bar{L} \cap C_{k}$ is a finite union of leaves. The proof of (4.0) is complete.

It is not true, in general, that every leaf of $\mathscr{F}$ pertains to a LMS. The alternative is described in the following easy corollary to (4.0) and will be analyzed in detail in $\$ 5$.

(4.8) CoRollary. A leaf $L$ of $\mathcal{F}$ belongs to a $L M S$ if and only if $\bar{L}$ is a finite union of LMS. In the alternative case, $\bar{L}$ contains LMS at arbitrarily high levels.

Proof. Since every LMS is at finite level, $L$ pertains to a LMS if and only if $\bar{L}=\bar{L} \cap M_{k}$ for $k$ sufficiently large. Such an equality holds if and only if $\bar{L}$ is a finite union of LMS. Suppose that $L$ does not pertain to a LMS and set $X_{k}=\bar{L} \cap$ $M_{k}, k \geqslant 0$. Let $U_{k}$ denote the component of $M-X_{k}$ containing $L$. By (3.0), $\bar{L} \cap U_{k}$ contains a LMS, necessarily at level $k+1$. That is, we obtain an infinite (proper) nest $X_{0} \subset X_{1} \subset \cdots \subset X_{k} \subset \cdots \subset \bar{L}$, so $\bar{L}$ contains LMS at arbitrarily high levels.

The following will also be useful.

(4.9) Corollary. Let $L$ be a totally proper leaf. Then every leaf of $\lim (L)$ has an element of contracting holonomy on whatever side is approached by $L$. 
Proof. Let $L^{\prime}$ be a leaf of $\lim (L)$ at level $k$. Let $J$ denote a compact, transverse arc $[y, z]$, properly crossing $L^{\prime}$, such that $J \cap \bar{L} \cap C_{k}=J \cap L^{\prime}=\{x\}$. This uses the fact that $\bar{L} \cap C_{k}$ contains only finitely many leaves (4.7). Indeed, $\bar{L}$ itself is a finite union of leaves by (4.7), so there must be a leaf $L^{\prime \prime}$ of $\bar{L}$ such that either $L^{\prime \prime} \cap(x, z]$ or $L^{\prime \prime} \cap[y, x)$ is a sequence $\left\{y_{m}\right\}$ converging monotonically to $x$ in $J$. By (3.4), some loop at $x$ on $L^{\prime}$ defines an element of holonomy $g$ such that $g\left(y_{m}\right) \neq y_{m}$ for some $m$. Then $g$ or $g^{-1}$ is the desired contraction.

5. Leaves at infinite level. Recall that a leaf $L$ is said to be at infinite level if it lies in no LMS. In this case, the substructure $S(L)$ is a union of leaves at arbitrarily high levels (4.8). This yields the first part of assertion (a) in (5.0). The remaining assertions of that theorem will now be proven in a series of lemmas.

(5.1) LEMMA. If $L$ is at infinite level, then $S(L)$ is dense in $\bar{L}$, but it contains no leaf that is dense in $\bar{L}$.

Proof. Let $S_{k}(L)=\bar{L} \cap M_{k}$, a compact, $\mathscr{F}$-saturated set (4.0). Since $L$ lies in no $M_{k}, S_{0}(L) \subset S_{1}(L) \subset \cdots \subset \bar{L}$, so $\cup_{k=0}^{\infty} S_{k}(L)=S(L)$ is dense in $\bar{L}$ by (4.2), but, of course, no $S_{k}(L)$ is dense in $\bar{L}$.

REMARK. Obviously, if $L$ is at infinite level, then no LMS in $\bar{L}$ can be of locally dense type. All must be either proper leaves or exceptional.

(5.2) LemMa. If $S(L)$ contains totally proper leaves at arbitrarily high levels, then every leaf of $S(L)$ is totally proper.

Proof. Let $Y$ denote the union of the totally proper leaves in $S(L)$. Applying (4.0) and (4.2) as in the proof of (5.1), we see that $Y$ is dense in $\bar{L}$. If $\bar{L}$ contains a LMS of exceptional type, let $L_{0}$ be a leaf in this LMS having an element $g$ of 2-sided contracting holonomy (cf. (2.2) and the remark following (2.3)). Since $L_{0} \subset \bar{L}=\bar{Y}$, some totally proper leaf meets the domain of $g$, hence is asymptotic to $L_{0}$. This contradiction implies that $Y=S(L)$.

Let $U_{k}$ denote the component of $M-S_{k}(L)$ containing $L$. Thus, $U_{k}$ is an open, connected, $\mathscr{F}$-saturated set for each $k \geqslant 0$.

(5.3) LEMMA. If $L$ is at infinite level, then there is an integer $N>0$ such that $\hat{U}_{k}$ is a foliated product for every $k \geqslant N$.

Proof. By (3.5), we need only show that $\cap_{k \geqslant 0} U_{k}$ has empty interior. If, on the contrary, $U$ is a nonempty component of $\operatorname{int}\left(\cap U_{k}\right)$, then $L \nsubseteq U$, by (5.1). Let $L_{1}, \ldots, L_{r}$ be the leaves of $\delta U$. We will prove that these leaves all lie in some $S_{k}(L)$. It will follow that $U$ is a component of $M-S_{k}(L)$ and, since $U \subseteq U_{k}$, we will have $U=U_{k}$, contradicting the fact that $L \nsubseteq U$.

Suppose, on the contrary, that at least one $L_{j}$ is not a leaf of any $S_{k}(L)$. It is easy to see that $L_{j} \subset \overline{S(L)}=\bar{L}$. If $L_{j}$ is proper, then $L_{j} \subseteq M_{k}$, for some $k$, and so $L_{j} \subseteq \bar{L} \cap M_{k}=S_{k}(L)$. Thus, $L_{j}$ is not proper. Since it has a proper side, $L_{j}$ is an exceptional leaf. Choose $x \in L_{j}$ and $x_{k} \in S_{k}(L)$ such that $\lim _{k \rightarrow \infty}\left(x_{k}\right)=x$ along an arc through $x$ in a leaf of $\mathcal{L}$. By (2.3) and the subsequent remark, there is a value 
of $k$ such that the leaf of $\mathscr{F}$ through $x_{k}$ is asymptotic to $L_{j}$. But $S_{k}(L)=\bar{L} \cap M_{k}$ is compact, so again $L_{j} \subseteq S_{k}(L)$ contrary to our assumption.

(5.4) LEMMA. If $L$ is at infinite level, then each leaf of $Z=\bar{L}-S(L)$ is dense in $\bar{L}$ and no leaf of $Z$ has a proper side.

Proof. Let $N \geqslant 0$ be as in (5.3). Let $k \geqslant N$, so $\hat{U}_{k}$ is a foliated product. Clearly, $Z \subset U_{k}$. Let $L_{*}$ be a leaf of $Z$. By (4.1), $\delta\left(U_{k}\right)$ is dense in $S_{k}(L)$; hence (3.2) implies that $L_{*}$ is asymptotic to every leaf of $S_{k}(L)-\delta\left(U_{k}\right)$. In fact, $L_{*}$ is asymptotic to every leaf of $S_{k}(L)$. To see this, fix $y \in L_{*}$ and let $J$ be the leaf of $\hat{\mathcal{L}}$ in $\hat{U}_{N}$ passing through $y$. Let $J_{i}$ be the component of $J \cap U_{i}$ containing $y, i \geqslant N$. Since $\cap_{i \geqslant N} U_{i}$ has empty interior (see the proof of (5.3)), the nest $\left\{J_{i}\right\}_{i>N}$ forms a fundamental neighborhood system of $y$ in $J$. Thus, there is $i>k$ such that $S_{k}(L) \subseteq S_{i}(L)-\delta\left(U_{i}\right)$. Since $L_{*}$ is asymptotic to every leaf of $S_{i}(L)-\delta\left(U_{i}\right)$, it is asymptotic to every leaf of $S_{k}(L)$. Since $k \geqslant N$ is arbitrary, $L_{*}$ is asymptotic to every leaf of $S(L)$; hence $\bar{L}_{*}=\overline{S(L)}=\bar{L}$. Also, $L_{*}$ approaches every leaf of $\delta\left(U_{k}\right)$, all $k \geqslant N$, and it follows that $L_{*}$ approaches itself from both sides.

(5.5) LemMa. If $L$ is at infinite level and $\mathscr{F}$ has smoothness class $C^{r}, 2<r<\infty$, then the holonomy of each leaf of $Z$ is $C^{r}$-tangent to the identity.

Proof. Let $L_{*}$ be a leaf of $Z$ and let $J$ and $y \in \operatorname{int}(J) \cap L_{*}$ be as in the proof of (5.4). For $k \geqslant N$, choose $x_{k} \in J \cap S_{k}(L)$ nearest to $y$ (hence, $x_{k} \in \delta\left(U_{k}\right)$ ). Then $\lim _{k \rightarrow \infty}\left(x_{k}\right)=y$ and every $x_{k}$ is a fixed point for the holonomy of $L_{*}$ at $y$. By iterated application of the mean value theorem, each holonomy element of $L_{*}$ is seen to be $C^{r}$-tangent to the identity.

RemarK. Examples show that a leaf at infinite level need not have trivial germinal holonomy.

(5.6) LEMMA. If $L$ is at infinite level, then $Z=\bar{L}-S(L)$ is an uncountable union of leaves.

Proof. Fix a leaf $J$ of $\hat{\mathfrak{L}}$ in $\hat{U}_{N}$. Each leaf of $Z$ meets the interior of $J$ in countably many points. We will show that $Z \cap J$ is uncountable. Let $y \in J \cap Z$ and let $J_{k}$ denote the leaf of $\hat{\mathcal{L}}$ in $\hat{U}_{k}$ containing $y, k>N$. Let $y^{\prime} \in J \cap Z$ lie on the same leaf of $\mathscr{F}$ as $y, y^{\prime} \neq y$. Then, for $k_{1}$ sufficiently large, $y^{\prime} \notin J_{k_{1}}$. There is a holonomy transformation $h$ in the foliated product $\hat{U}_{N}$ such that $y^{\prime} \in h\left(J_{k_{1}}\right)$. Since $\operatorname{dom}(h)=J$, the iterates of $h$ move $J_{k_{1}}$ to a countably infinite set of intervals. Thus, $\hat{U}_{k_{1}}$ meets $J$ (and, indeed, each leaf of $\hat{\mathfrak{L}}$ in $\hat{U}_{N}$ ) in countably many distinct intervals. By the same method, applied inductively, we find integers $\left\{k_{m}\right\}_{m>1}$ such that $\hat{U}_{k_{m+1}} \subset U_{k_{m}}$ and $\hat{U}_{k_{m+1}}$ meets each of the countably many intervals of $J \cap \hat{U}_{k_{m}}$ in countably many intervals.

Consider the set 9 of all sequences $\left\{I_{m}\right\}_{m>1}$ where each $I_{m}$ is a component of $\hat{U}_{k_{m}} \cap J$ and $I_{1} \supset I_{2} \supset \cdots \supset I_{m} \supset \cdots$. Since $\cap_{m>1} U_{k_{m}}$ has empty interior, it follows that $\cap_{m \geqslant 1} I_{m}$ is a single point of $Z \cap J$. This sets up a one-one correspondence between $G$ and $Z \cap J$. By the above, it is evident that $G$ is an uncountable set.

All assertions in (5.0) have been established. 
6. The Poincaré-Bendixson theory of totally proper leaves. So far we have been concerned with the structure of $\lim (L)$ where $L$ is an arbitrary leaf of $\mathscr{F}$. Much stronger results are available when $L$ has nonexponential growth (7.0), the totally proper leaves in $\lim (L)$ playing a particularly important role.

In the present section, which we consider to be the real core of the paper, we will analyze the way in which totally proper leaves "spiral in" on leaves at lower levels. This generalizes the Poincaré-Bendixson theory of nowhere dense leaves in $C^{2}$-foliations of $T^{2}$ [Sc] and will yield, in particular, a proof of (6.0).

Shortly, we will define the "spiraling" mentioned above, but first we indicate examples which may make the definition more understandable.

In Figure la, we depict a part $B_{i}$ of a (closed) neighborhood $B$ (in a 2-dimensional leaf) of a planar end at level 0 (cf. §1) that spirals on a toral leaf and, in Figure 1b, the entire neighborhood $B=\cup B_{i}$ is indicated. (An end $e$ of an orientable surface $N$ is called planar if it has a neighborhood $B$ in $N$ such that $\operatorname{int}(B)$ is homeomorphic to an open subset of $\mathbf{R}^{2}$. Otherwise, $e$ is nonplanar.) The behavior in Figure 1, of course, is exemplified in the Reeb foliation.

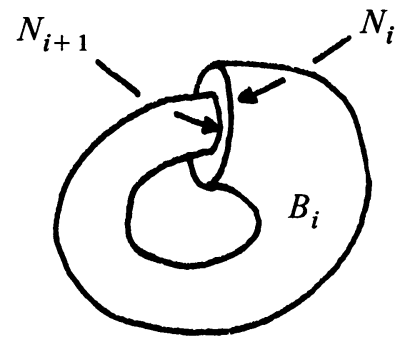

(a)

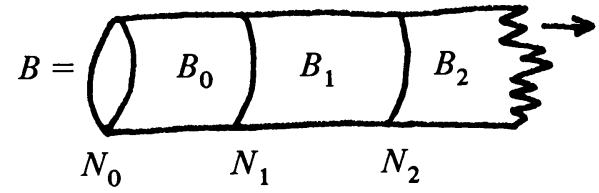

(b)

FIGURE 1

Similarly, in Figure $2 \mathrm{a}$, a part $\boldsymbol{B}_{\boldsymbol{i}}$ of a neighborhood $\boldsymbol{B}$ of a nonplanar end at level 0 that spirals in on a 2 -holed toral leaf is shown, and $B$ itself is pictured in Figure $2 b$.

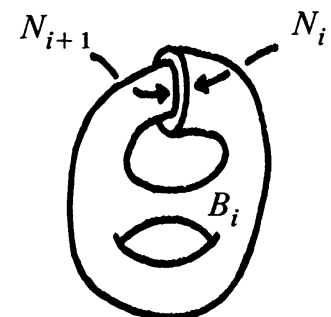

(a)

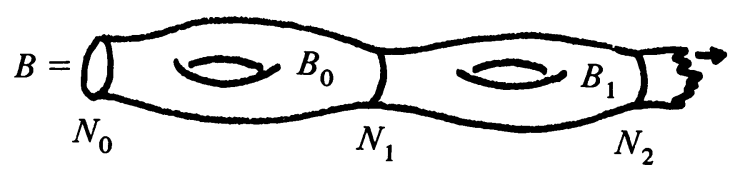

(b)

FIGURE 2 
Finally, in Figure 3 we show a neighborhood $B=\cup B_{i}$ of a planar end at level 1 which spirals in on a leaf $L_{0}$ at level 1 . In Figure 3c, the leaf $L_{0}$ is also pictured. Note that the two ends of $L_{0}$ spiral in on toral leaves and that the ends of $B$ at level 0 also spiral in on these tori.

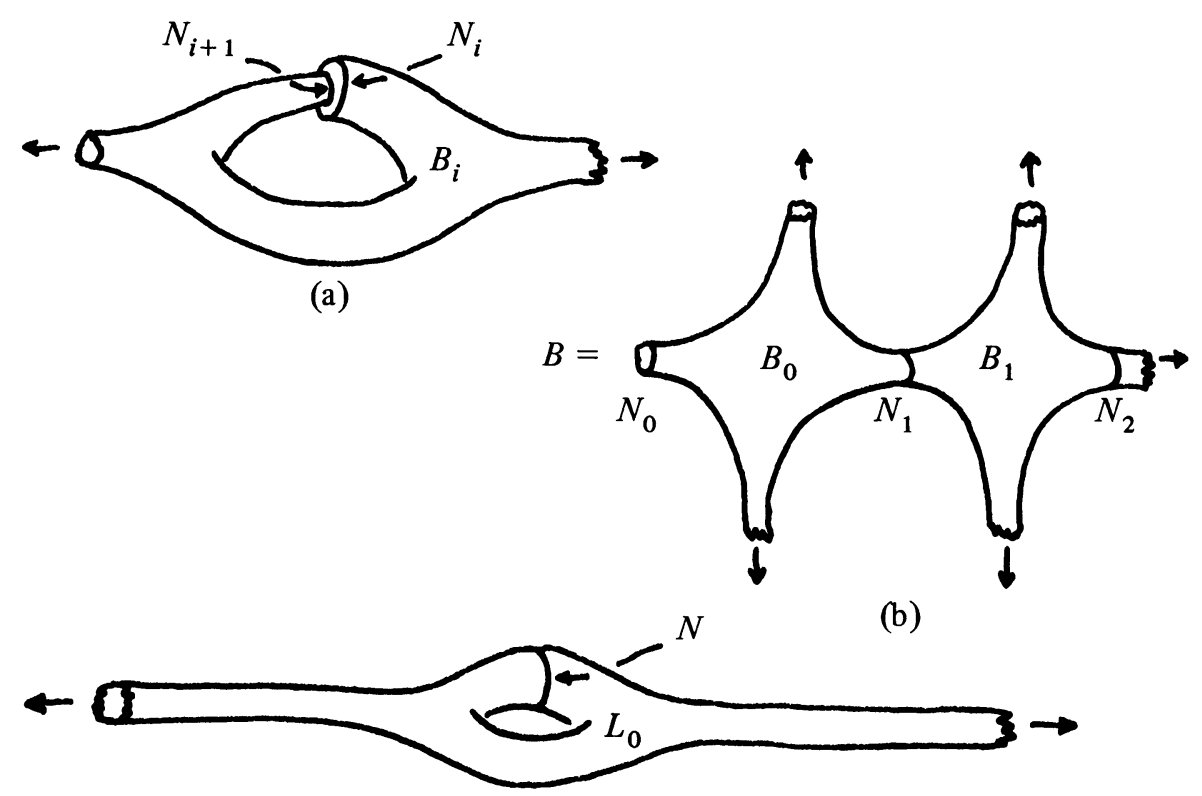

(c)

\section{Figure 3}

Definition. Let $\left(x_{0}, x\right]$ be a subarc of a leaf of $\mathcal{L}$, and let $L_{0}$ be the leaf of $\mathscr{F}$ through $x_{0}$. If $\left(x_{0}, x\right] \cap L_{0}=\varnothing$ we say that $x$ projects (in the negative direction) to $L_{0}$ and we write $p(x)=x_{0} \in L_{0}$.

Let $L$ and $L_{0}$ be leaves of $\mathcal{F}$ and let $B \subset L$ be a complete connected, noncompact submanifold of dimension $n-1$. Suppose that $N_{0}=\partial B$ is a compact, connected manifold. Finally, suppose that each point of $B$ projects to $L_{0}$ as above and remark that $p: B \rightarrow L_{0}$ is locally a diffeomorphism. The following generalizes ideas in [Sc], [So], and [Ni].

Definition. We will say that $B$ spirals on $L_{0}$ (on the positive side) if the following conditions are satisfied.

(a) $B=\cup_{i=0}^{\infty} B_{i}$ where each $B_{i}$ is a complete connected, $(n-1)$-dimensional submanifold, $\partial B_{i}=N_{i} \cup N_{i+1}$ is a union of two compact components, and $\operatorname{int}\left(B_{i}\right)$ $\cap \operatorname{int}\left(B_{j}\right)=\varnothing$ if $i \neq j$

(b) there is a compact, connected, ( $n-2)$-dimensional submanifold $N \subset L_{0}$ (called the juncture of the spiral) such that $p \mid N_{i}$ maps $N_{i}$ diffeomorphically onto $N$, $0 \leqslant i<\infty$;

(c) for each $y \in L_{0}$ and each $i \geqslant 0, p^{-1}(y) \cap\left(B_{i}-N_{i+1}\right)$ is a single point $y_{i}$;

(d) for each $y \in L_{0}$, the sequence $p^{-1}(y)=\left\{y_{i}\right\}_{i>0}$ converges monotonically to $y$ in $\left[y, y_{0}\right]$. 
Similarly, projection in the positive direction and spirals on the negative side of $L_{0}$ are defined.

Remark that $\operatorname{int}\left(B_{i}\right) \cong L_{0}-N, i \geqslant 0$. In particular, $N$ does not separate $L_{0}$. Also remark that $\left\{B(k)=\cup_{i \geqslant k} B_{i}\right\}_{k \geqslant 0}$ is a fundamental neighborhood system of an end $e \in \mathcal{E}(L)$.

Definition. The end $e \in \mathcal{E}(L)$ as above is said to be periodic of period $L_{0}$.

We can now state the basic result of this section. We will postpone its proof, first deriving (6.0) as a consequence.

(6.1) TheOREM. Let $L$ be a leaf with a totally proper end $e$ at level $k$. Then $e$ is periodic of period $L_{0}$, where $L_{0}$ is totally proper at level $k$.

In particular, if $L$ is totally proper, so are all of its ends, and we will use (6.1) to decompose $L$ into a compact piece and finitely many spirals. With this, (6.0) will be an elementary consequence.

(6.2) Lemma. Let $L$ be a totally proper leaf of $\mathcal{F}$. Let $\left\{e_{m}\right\} \subset \mathcal{E}(L)$ be a sequence converging to $e \in \mathcal{E}(L)$, and suppose that $e$ is at level $k$. Then, for $m$ sufficiently large, $e_{m}$ is at level strictly less than $k$.

Proof. Otherwise, we can choose all $e_{m}$ to be at the same level $\geqslant k$. Since $\bar{L}$ is a finite union of leaves (4.7), we can assume that all $e_{m}$ are asymptotic to a common leaf $L^{\prime} \subset \lim (L)$ at level at least $k$. Without loss of generality, let each $e_{m}$ be asymptotic to $L^{\prime}$ on the positive side (where we do not rule out, a priori, that $e_{m}$ might be asymptotic to $L^{\prime}$ on both sides), fix $x \in L^{\prime}$ and a transverse $\operatorname{arc}(x, z]$ not meeting $\bar{L} \cap C_{k}$. By (4.9), there is a loop $\sigma$ on $L^{\prime}$ at $x$ defining an element $f$ of contracting holonomy, and we can assume $\operatorname{dom}(f)=(x, z]$. In $L$, choose an expanding nest of compact sets $K_{1} \subset K_{2} \subset \cdots, L=\cup K_{m}$, and a fundamental system $U_{1} \supset U_{2} \supset \cdots$ of open neighborhoods of $e$ such that each $U_{m}$ is a component of $L-K_{m}$ and both $U_{m}$ and $U_{m}-U_{m+1}$ are neighborhoods of $e_{m}$. Since $U_{m}$ is a component of $L-K_{m}$, where $K_{m}$ is compact, we can find $v_{m} \downarrow x$ in $(x, z]$ such that, for every $y \in\left(x, v_{m}\right], \sigma$ lifts to a path $\tilde{\sigma}$ on the leaf through $y$ and $\tilde{\sigma} \cap K_{m+1}=\varnothing$. Since $L^{\prime} \subset e_{m}-\lim (L) \subset \overline{\left(U_{m}-U_{m+1}\right)}$, choose $y_{m} \in\left(x, v_{m}\right] \cap\left(U_{m}\right.$ $\left.-U_{m+1}\right)$. Then the sets $\left\{f^{i}\left(y_{m}\right)\right\}_{i>0}$ and $\left\{f^{i}\left(y_{r}\right)\right\}_{i>0}$ are disjoint if $m \neq r$. Let $p_{m} \geqslant 0$ be such that $x_{m}=f^{-p_{m}}\left(y_{m}\right) \in[f(z), z]$. If $x_{m}=x_{r}$, then we claim $m=r$. Indeed, choose $p>\max \left\{p_{m}, p_{r}\right\}$ and observe that $f^{p-p_{m}}\left(y_{m}\right)=f^{p-p_{r}}\left(y_{r}\right)$, so $m=r$. Thus there is $w \in[f(z), z]$ at which $\left\{x_{m}\right\}_{m>1}$ clusters. The leaf $L^{\prime \prime}$ through $w$ belongs to $\lim (L)$ and accumulates on $L^{\prime}$; hence $L^{\prime \prime}$ must be totally proper at level $\geqslant k+1$. If we can show $L^{\prime \prime} \subset e-\lim (L)$, we will have the desired contradiction. For a fixed but arbitrary positive integer $m$, choose $N>0$ so large that $f^{N}(z) \in\left(x, v_{m}\right]$. Since also $y_{r} \in\left(x, v_{m}\right] \cap U_{m}$ for $r \geqslant m$, we see that $f^{N}\left(x_{r}\right)=$ $f^{N-\operatorname{Pr}}\left(y_{r}\right)$ is a point of $U_{m}$. That is, $f^{N}\left(x_{r}\right) \in\left[f^{N+1}(z), f^{N}(z)\right] \cap U_{m}$, all $r>m$, so $U_{m}$ accumulates at $f^{N}(w) \in L^{\prime \prime}$. Since $m$ is arbitrary, $L^{\prime \prime} \subset e-\lim (L)$.

(6.3) Proposition. If $L$ is a totally proper leaf at level $k$, then $L=A \cup B^{1}$ $\cup \cdots \cup B^{r}$, where $A$ is a compact, connected $(n-1)$-dimensional manifold with 
boundary components $N^{1}, \ldots, N^{r}$ and

(a) $A \cap B^{j}=N^{j}, 1 \leqslant j \leqslant r$;

(b) $B^{i} \cap B^{j}=\varnothing, i \neq j$;

(c) each $B^{j}$ spirals on a totally proper leaf $L_{j}$ at level at most $k-1$;

(d) for at least one value of $j, L_{j}$ is at level $k-1$.

Proof. By (6.2), $L$ has at most finitely many ends $e_{1}, \ldots, e_{r(1)}$ at level $k-1$. Since $\lim (L)$ is the union of all $e-\lim (L), e \in \mathcal{E}(L), L$ does have at least one end at level $k-1$. By (6.1), each $e_{i}$ has a closed neighborhood $B^{i} \subset L$ spiraling on a totally proper leaf at level $k-1$. We can arrange that $B^{i} \cap B^{j}=\varnothing, i \neq j$. Let $N^{i}=\partial B^{i}$, a compact, connected, $(n-2)$-dimensional manifold. It is elementary that one can produce a compact, connected, $(n-1)$-dimensional submanifold $A \subset L-\cup_{i=1}^{r(1)} \operatorname{int}\left(B^{i}\right)$ such that $\cup_{i=1}^{r(1)} N^{i} \subseteq \partial A$. Enlarging $A$, if necessary, one also arranges that $L-\operatorname{int}(A)$ has no compact components. Since $\partial A$ has only finitely many components, there are at most finitely many components $B^{j}, r(1)<j$ $\leqslant s$, of $L-\operatorname{int}(A)$ other than the components $B^{i}, 1<i<r(1)$. Each $\mathcal{E}\left(B^{j}\right)$, $j>r(1)$, contains at most a finite number of ends at level $k-2$ (again by (6.2)) and we apply (6.1) to each of these, enlarging $A$ and increasing $s$, if necessary, to obtain

$$
L=A \cup B^{1} \cup \cdots \cup B^{r(1)} \cup B^{r(1)+1} \cup \cdots \cup B^{r(2)} \cup \cdots \cup B^{s}
$$

where, for $r(1)<j \leqslant r(2), B^{j}$ spirals on a totally proper leaf at level $k-2$. Here we allow $r(1)=r(2)$ in case there are no ends at level $k-2$ in $\mathcal{E}\left(B^{j}\right), j>r(1)$. Finite repetition of this process produces a decomposition of $L$ as desired.

We begin the proof of (6.0). By [C-C3, Proposition 3], a nowhere dense leaf with growth dominated by a polynomial will have to be totally proper. (By [C-C3, Theorem 4], it will also be a manifold of finite type.) Thus, we must show that every totally proper leaf at level $k$ has exactly polynomial growth of degree $k$ and also has type $k-1$.

First we consider the question of type. A totally proper leaf at level 0 is compact; hence it has type -1 . Inductively, suppose that totally proper leaves at level $q<k$ have type $q-1$ and let $L$ be a totally proper leaf at level $k+1$. Write $L=A \cup$ $B^{1} \cup \cdots \cup B^{r}$ as in (6.3). Since $B^{i}$ spirals on a leaf at level $q<k$, the fact that this leaf has type $q-1$ readily shows that the endset $\mathscr{E}\left(B^{i}\right)$ has type $q<k$ (indeed, $\mathcal{E}^{(q)}\left(B^{i}\right)$ is a singleton). Since $\mathcal{E}(L)$ is the disjoint union of the open-closed subsets $\mathcal{E}\left(B^{i}\right), 1 \leqslant i \leqslant r$, and since $q=k$ for at least one $B^{i}$, it follows that $L$ is a manifold of type $k$.

Next we consider the growth. Again, it is obvious that totally proper leaves at level 0 have exactly polynomial growth of degree 0 . Inductively, suppose that totally proper leaves at level $q \leqslant k$ have exactly polynomial growth of degree $q$ and let $L$ be a totally proper leaf at level $k+1$. Write $L=A \cup B^{1} \cup \cdots \cup B^{r}$ as in (6.3). One defines the growth type of each $B^{i}$ exactly as for the whole leaf $L$. If $B^{i}$ has exactly polynomial growth of degree $a_{i}, 1 \leqslant i<r$, then $L$ has exactly polynomial growth of degree $a=\max \left\{a_{i}\right\}$. Because of the inductive hypothesis, it will be sufficient to show that, if $B^{i}=B=\cup_{j>0} B_{j}$ spirals on a leaf $L_{0}$ and if $L_{0}$ has 
exactly polynomial growth of degree $q$, then $B$ has exactly polynomial growth of degree $q+1$.

Let $N \subset L_{0}$ be the juncture and let $N(\varepsilon) \cong N \times[-\varepsilon, \varepsilon]$ be a normal neighborhood of $N$ in $L_{0}$. Let $N_{j}(\varepsilon)$ be a normal neighborhood of $N_{j}$ in $B, j>1$, projecting diffeomorphically onto $N(\varepsilon)$, and let $N_{0}(\varepsilon) \cong N_{0} \times[0, \varepsilon]$ be the normal half-neighborhood in $B$. Evidently, we can produce an imbedding $\varphi_{0}: N(\varepsilon) \times I \rightarrow M$, $W_{0}=\operatorname{Im}\left(\varphi_{0}\right)$, such that $W_{0} \cap L_{0}=N(\varepsilon)$ and $W_{0} \cap B=\cup_{j>0} N_{j}(\varepsilon)$. Construct an $(\mathscr{F}, \mathcal{L})$ coordinate atlas on $M$ (see $\S 3$ ) such that $W_{0}$ is a union of charts and such that the remaining charts $\left\{\left(W_{i}, \varphi_{i}\right)\right\}_{i=1}^{m}$ satisfy $W_{i} \cap N_{j}=\varnothing=W_{i} \cap N$, $j \geqslant 0$. Number these so that $W_{i} \cap L_{0} \neq \varnothing$ exactly for $1<i<s$. We can assume that $W_{i}$ meets each $B_{j}, j \geqslant 0$, for $1 \leqslant i \leqslant s$. Although $\left(W_{0}, \varphi_{0}\right)$ is not an $(\mathscr{F}, \mathcal{E})$ chart, it serves as one relative to $B$ and $L_{0}$ with "plaques" $N_{j}(\varepsilon)$ and $N(\varepsilon)$.

As in [P1, §4], the growth types of $L_{0}, B$, and $B_{j}$ can be measured in terms of the plaques of $W_{i}, 0 \leqslant i \leqslant s$. Let $P_{0}=W_{0} \cap L_{0}=N(\varepsilon)$. Let $g_{0}(l)$ be the number of distinct plaques on $L_{0}$ that can be reached by chains of plaques $P_{0}, P_{1}, \ldots, P_{r}$, $r \leqslant l$, where $P_{i} \cap P_{i+1} \neq \varnothing, 0 \leqslant i \leqslant r-1$. The growth type of $g_{0}$ coincides with that of the leaf $L_{0}$.

Similarly, let $P_{0, j}=N_{j}(\varepsilon), j \geqslant 0$. Let $g(l)$ denote the number of plaques meeting $B$ that can be reached by chains $P_{0,0}, P_{1}, \ldots, P_{r}, r<l$, and let $h_{j}(l)$ denote the analogous function for $B_{j}$ and chains $P_{0_{j}}, P_{1}, \ldots, P_{r}$. As we see by projection, $h_{j}(l)$ is independent of $j$, so we will denote this function by $h(l)$.

Since $N$ is compact and does not disconnect $L_{0}, L_{0}-N$ has the same growth type as $L_{0}$. Letting $P_{*}=p\left(N_{0}(\varepsilon)\right) \cong N \times[0, \varepsilon]$, define $g_{*}(l)$ for $L_{0}-N$ via chains $P_{*}, P_{1}, \ldots, P_{r}, r \leqslant l$. Again, by projection, it is evident that $g_{*}(l)=h(l)$.

Let $n_{0}$ be the smallest integer such that there is a chain $P_{0,0}, P_{1}, \ldots, P_{n_{0}-1}, P_{0,1}$ for $B_{0}$. The same integer $n_{0}$ plays the analogous role for each $B_{j}, j \geqslant 0$. By the division algorithm, write $l=\lambda n_{0}+r$ and note that $g(l)=h(l)+h\left(l-n_{0}\right)+$ $h\left(l-2 n_{0}\right)+\cdots+h(r)$.

Since we assume that $L_{0}$ has exactly polynomial growth of degree $q$, we see that $h=g_{*}$ has the growth type of a polynomial of degree $q$. By the above formula for $g, g(l) \leqslant h(l)+h(l-1)+\cdots+h(1) \leqslant \operatorname{lh}(l)$ and

$$
\begin{aligned}
g(2 l) & \geqslant h(2 l)+h\left(2 l-n_{0}\right)+\cdots+h\left(2 l-(\lambda-1) n_{0}\right) \\
& \geqslant h(l)+h(l)+\cdots+h(l) \quad(\lambda \text { terms }) \\
& =\left(1 / n_{0}\right)(l-r) h(l)>\left(1 / n_{0}\right)\left(l-n_{0}\right) h(l) .
\end{aligned}
$$

That is, $g$ has the growth type of a polynomial of degree $q+1$, and this is also the growth type of $B$. The proof of (6.0) is complete.

We turn to the proof of (6.1).

First, remark that if $B$ is a complete, connected, noncompact, $(n-1)$ dimensional submanifold of a leaf $L$ and if $\partial B$ is compact, then $\lim (B)$ can be defined exactly as for a leaf and it is a nonempty, compact, $\mathscr{F}$-saturated set.

(6.4) LemMA. Let $L$ be a leaf with a totally proper end $e$ at level $k$. Then $e$ has a (closed) neighborhood $\tilde{B} \subset L$ such that $\lim (\tilde{B})=e-\lim (L)$ and such that $\tilde{B}$ is not a neighborhood of any other end at level $k$. 
Proof. If, for every closed neighborhood $\tilde{B}$ of $e, \lim (\tilde{B}) \neq e-\lim (L)$, we can choose a fundamental system of closed neighborhoods $L \supset B_{1} \supset B_{2} \supset \cdots$ of $e$ and leaves $L_{i} \subseteq \lim \left(B_{i}\right)-(e-\lim (L))$. We do not claim $L_{i} \neq L_{j}$ for $i \neq j$. In $B_{i}$, choose $\left\{x_{i j}\right\}_{j=1}^{\infty}$ converging to $x_{i} \in L_{i}$. We can assume that $\left\{x_{i}\right\}$ converges to $x \in M$ and use the diagonal process to choose $\left\{y_{i}=x_{i, j(i)}\right\}_{i=1}^{\infty}$ also converging to $x$. In $L \cup \mathcal{E}(L), y_{i} \rightarrow e$ and so $x \in e-\lim (L)$. In particular, $x \notin L_{i}, i \geqslant 1$. Let $L^{\prime}$ be the leaf of $\mathscr{F}$ through $x$, necessarily at level $k^{\prime}<k$. Choose $(x, z]$ to meet no leaf at level $\leqslant k^{\prime}$. Having possibly reversed the transverse orientation of $\mathscr{F}$ and/or passed to a subsequence of $\left\{L_{i}\right\}$, we can assume that, for arbitrary $b \in(x, z], L_{i} \cap(x, b]$ $\neq \varnothing$ for $i$ sufficiently large. Choose $[c, b] \subset(x, z]$ such that every leaf of $\mathscr{F}$ that meets $(x, b]$ also meets $(c, b)$. This can be done using (3.8), the holonomy along $L^{\prime}$ being unbounded by (3.4) and the fact that $L^{\prime} \subset e-\lim (L)$. Then, for $i$ sufficiently large, one can choose $z_{i} \in L_{i} \cap[c, b]$. By passing to a subsequence, we obtain $w=\lim \left(z_{i}\right) \in[c, b]$. Choosing $\left\{z_{i j}\right\}_{j=1}^{\infty} \subset B_{i}$ converging to $z_{i}$ in $M$, and again using the diagonal process, we see that the leaf $L^{\prime \prime}$ through $w$ lies in $e-\lim (L)$ and is at level $k^{\prime \prime}>k^{\prime}$. A finite iteration of this procedure will produce a leaf of $e-\lim (L)$ at level greater than $k$, a contradiction.

Thus, we find $\tilde{B}$ such that $\lim (\tilde{B})=e-\lim (L)$. Suppose $\tilde{B}$ is a neighborhood of infinitely many ends at level $k$. By $(4.0), \lim (\tilde{B})$ is a finite union of totally proper leaves and, by the same arguments as in the proof of $(4.9)$, each leaf of $\lim (\tilde{B})$ has an element of contracting holonomy on whatever side is approached by $\tilde{B}$. Thus, the argument in the proof of (6.2) applies and shows that $\tilde{B}$ must be a neighborhood of an end at level greater than $k$, contradicting the fact that $\lim (\tilde{B})=e$ $\lim (L)$. Thus, we can choose $\tilde{B}$ small enough to exclude all ends at level $k$ other than $e$.

Fixing $\tilde{B}$ as in (6.4), we will find $B \subseteq \tilde{B}$, also a closed neighborhood of $e$, and a leaf $L_{0}$ at level $k$ such that $B$ spirals on $L_{0}$. This will complete the proof of (6.1).

Let $U$ be the component of $M-(e-\lim (L))$ containing $L$. There is a leaf $L_{0} \subset \delta U \subseteq e-\lim (L)$ that is (totally proper) at level $k$. We suppose that $\tilde{B}$ approaches $L_{0}$ on the positive side and select $x \in L_{0}$ and a transverse $\operatorname{arc}(x, z] \subset U$. Since $\lim (\tilde{B})=e-\lim (L)$, we see that $\tilde{B} \cap(x, z]=\left\{y_{m}\right\}_{m=1}^{\infty}$ is a sequence converging monotonically to $x$. We can assume that $y_{m} \notin \partial \tilde{B}$, for all $m>1$.

Decompose $L_{0}=A \cup B^{1} \cup \cdots \cup B^{r}$ so as to satisfy (3.3) relative to $\hat{U}, x \in$ $\operatorname{int}(A)$. In the following, it may be necessary to rechoose $A$ larger. This may multiply the number of "arms" $B^{i}$, but each of these will be in one or another of the original arms.

Let $G_{0}$ be a finite set of loops at $x$ generating $\pi_{1}(A, x), G_{i}$ a set of loops in $B^{i}$ based at $x_{i} \in \partial B^{i}, 1 \leqslant i \leqslant r$, generating $\pi_{1}\left(B^{i}, x_{i}\right)$, fix paths $\tau_{i}$ in $A$ from $x$ to $x_{i}$, and regard $G=G_{0} \cup \tau_{1}^{-1} G_{1} \tau_{1} \cup \cdots \cup \tau_{r}^{-1} G_{r} \tau_{r}$ as a set of generators for $\pi_{1}\left(L_{0}, x\right)$.

(6.5) LEMMA. If $A$ is chosen sufficiently large, there is an integer $K>0$ such that, for each $\sigma \in G$, the holonomy transformation $h_{\sigma}$ and $/$ or $h_{\sigma}^{-1}$, defined by $\sigma$, maps the set $\left\{y_{m}\right\}_{m>K}$ into itself. Furthermore, there is some $\sigma \in G, \varepsilon= \pm 1$, and an integer $\rho>0$ such that $h_{\sigma}^{e}\left(y_{m}\right)=y_{m+\rho}$ for all $m>K$. In particular, this $h_{\sigma}^{e}$ is an element of contracting holonomy. 
Proof. Let $W \subseteq U$ be an open (not necessarily $\mathscr{F}$-saturated) set such that, for each $w \in W, p(w) \in L_{0}$ is defined and the arc $(p(w), w]$ lies in $W$. Furthermore, if $[\alpha, \beta]$ is any leaf of the $\hat{\mathcal{L}}$-saturation of $B^{i}$ in $\hat{U}, 1 \leqslant i \leqslant r$, require that $(\alpha, \beta) \subset$ $W$. Let $X=W \cap \partial \tilde{B}$ and remark that $p(X)$ is a relatively compact subset of $L_{0}$. Thus, without loss of generality, assume that $p(X) \subset \operatorname{int}(A)$. It is then possible to choose $W$ satisfying all of the above so that $W \cap \partial \tilde{B}=\varnothing$. For a suitable integer $K>0$, every $\sigma \in G$ lifts to a path on $L \cap W$ starting at $y_{m}, \forall m \geqslant K$, and such lifts actually lie in $\tilde{B} \cap W$ since $W \cap \partial \tilde{B}=\varnothing$. This is a consequence of the finiteness of $G_{0}$ and of the product structure of the $\hat{\mathcal{L}}$-saturation of $B^{i}, 1<i<r$. For $m \geqslant K$ and for each $\sigma \in G$, there is $\sigma_{*}(m) \in \mathbf{Z}$ such that $h_{\sigma}\left(y_{m}\right)=y_{\sigma_{*}(m)}$. If $\sigma_{*}(K)=K$, for all $\sigma \in G$, then in standard fashion we find a subset $L^{*}$ of $\tilde{B}$ projecting diffeomorphically onto $L_{0}$. But then $L^{\prime}=L$ and $L$ cannot approach $L_{0}$. Thus, we can find $\sigma \in G$ and $\varepsilon= \pm 1$ such that $h_{\sigma}^{e}\left(y_{K}\right)=y_{K+\rho}$ for some $\rho>0$. It follows that $h_{\sigma}^{e}\left(y_{m}\right)=y_{m+\rho}$, for every $m \geqslant K$.

ReMarK. If, for some $\sigma \in G, h_{\sigma}\left(y_{K}\right)=y_{K}$, then $h_{\sigma}\left(y_{m}\right)=y_{m}$, for all $m \geqslant K$.

(6.6) LEMMA. The decomposition $L_{0}=A \cup B^{1} \cup \cdots \cup B^{r}$ and the associated generating set $G$ can be chosen so that, if $\sigma \in G-G_{0}$, then $h_{\sigma}\left(y_{m}\right)=y_{m}$, for all $m \geqslant K$.

Proof. As in the proof of (3.9), the holonomy pseudogroup of $\mathscr{F}$, defined relative to a suitable biregular cover, defines a finitely generated, transitive permuter pseudogroup of the system $g_{i}$ of intervals defined by the $\hat{\mathfrak{L}}$-saturation of $B^{i}$, $1 \leqslant i \leqslant r$. In the generalized Kopell lemma (2.8), take $J=J_{i}$ to be the $\hat{\mathcal{L}}$-leaf at $x_{i}$ and take $b=b_{i}=h_{\tau_{i}}\left(y_{K}\right), a=a_{i}=h_{\tau_{i}}\left(y_{K+1}\right)$ (defined if $K$ is chosen large enough). An obvious application of (2.8) then allows us to choose $A$ larger so that, for each $i$, the holonomy group on $J_{i}$ defined by $\pi_{1}\left(B^{i}, x_{i}\right)$ is generated by elements $\gamma$ (basic loops at $J_{i}$ ) such that $a_{i}<\gamma\left(b_{i}\right)$. But this implies that $\gamma\left(b_{i}\right)=b_{i}$, hence that $h_{\sigma}\left(y_{K}\right)=y_{K}, \forall \sigma \in G_{i}, \forall i$.

We fix the decomposition of $L_{0}$ as in (6.6). Let $\lambda$ be the smallest positive integer such that $h_{\sigma}\left(y_{K}\right)=y_{K+\lambda}$, for some loop $\sigma$ in $A$ based at $x$. Define $\eta: \pi_{1}(A, x) \rightarrow \mathbf{Z}$ by $h_{\sigma}\left(y_{m}\right)=y_{m+\eta(\sigma) \lambda}$, for $m$ large. This is a surjective homomorphism; hence it defines a surjection $\eta: H_{1}(A) \rightarrow \mathrm{Z}$ and $\eta$ can be regarded as a nondivisible class in $H^{1}(A)$. Let $f: A \rightarrow S^{1}$ be a classifying map for $\eta$. That is, if $\theta \in H^{1}\left(S^{1}\right)$ is the fundamental class, then $\eta=f^{*}(\theta)$.

Let $i: \partial A \hookrightarrow A$ be the inclusion. A connected cycle $c$ on $\partial A$ is homologous to an integral linear combination of loops in some $G_{j}$; hence, by $(6.6), \eta(c)=0$. That is, $i^{*}(\eta)=0$ and this implies that $f \mid \partial A$ is inessential. Therefore, $f$ is homotopic to a smooth map $g: A \rightarrow S^{1}$ having a regular value $p_{0} \notin g(\partial A)$. Let $N=g^{-1}\left(p_{0}\right)$, a compact, orientable, $(n-2)$-dimensional submanifold of $A$ with $\partial N=N \cap \partial A=$ $\varnothing$. This manifold represents the Poincaré dual $\eta_{*} \in H_{n-2}(A, \partial A)$ of $\eta$. Since $\eta_{*}$ is nondivisible, $N$ is homologous to a connected manifold, so we can assume that $N$ is connected.

In the choice of $G_{0} \subset G$ generating $\pi_{1}(A, x)$ we select $\tau, \sigma^{1}, \ldots, \sigma^{m}$ so that the intersection number of $\tau$ with $N$ is 1 and that of each $\sigma^{i}$ with $N$ is 0 , we conclude 
from the above that $h_{\tau}$ is a contraction with $h_{\tau}\left(y_{j}\right)=y_{j+\lambda}$ and that $h_{\left(\sigma^{i}\right)}\left(y_{j}\right)=y_{j}$, for large enough $j$. Fix such $j_{0} \geqslant K$. Define $B_{j} \subset \tilde{B}$ to be the set of points that can be reached from $y_{j_{0}+j \lambda}$ by the lifts of paths on $L_{0}$ starting at $x$ and issuing from the same side of $N$ as does $\tau$, but with interiors not meeting $N$. Evidently, for each $j \geqslant 0, B_{j}$ has exactly two boundary components, $N_{j}$ and $N_{j+1}$, each mapped diffeomorphically onto $N$ by $p$, and $p: B_{j}-\partial B_{j} \rightarrow L_{0}-N$ is also a diffeomorphism. Since $\left\{y_{j}\right\}$ converges monotonically to $x$ in $[x, z]$, it follows that $B=B_{0} \cup$ $B_{1} \cup \cdots$ spirals on $L_{0}$. As such, it is a neighborhood of at least one totally proper end at level $k$. Since $\tilde{B}$ is a neighborhood of only one such end, namely $e$, it follows that $B$ is also a neighborhood of $e$. (It also follows that $\lambda=1$, but we do not need that fact.)

We have finally established (6.1).

7. Leaves with nonexponential growth. We give here some corollaries to the basic structure theorem (7.0) for nonexponential leaves. As remarked in the introduction, (7.0) itself has already been proven in [C-C4] by use of the other results of this paper.

First we remark on the various possibilities allowed in (7.0). In case (a), (6.0) asserts that the leaf must be exactly polynomial and that it must be a manifold of finite type. In [C-C1], it was shown that "almost all" orientable surfaces of finite type actually occur as totally proper leaves in suitable, $C^{\infty}$-foliated 3 -manifolds. In case (b), examples [C-C4] show that $L$ need not have exactly polynomial growth. Indeed, there is a continuum of growth types possible, each dominating all polynomial types. Furthermore, we can show that the "fractional growth" examples in [C-C4] can be made $C^{2}$ if they are required to dominate the cubic type, so this provides a continuum of distinct growth types in case (b), all dominated by polynomials. If a nonexponential leaf is at infinite level, it cannot have growth dominated by a polynomial [C-C3, Proposition 2], and examples show that continuously many growth types, all occurring in the same foliation, are available [He]. It is also possible for a leaf at infinite level to have exponential growth [He] and for an exponential leaf at finite level to have totally proper substructure and to satisfy (b) of (7.0) [C-C4].

(7.1) COROllaRY. If $L$ is a leaf of $\mathcal{F}$ with neither exponential nor exactly polynomial growth, then the holonomy of $L$ is either germinally trivial or it is $C^{r}$-tangent to the identity (where $\mathcal{F}$ is of class $C^{r}, 2<r<\infty$ ).

This is an immediate consequence of (5.0), (6.0), and (7.0).

(7.2) COROLlaRY. If $L$ is a leaf of $\mathscr{F}$ having growth dominated by the polynomial $x^{k}$, then the leaves of $\bar{L}$ all have growth dominated by this same polynomial.

Proof. If $L$ is totally proper, this is clear. Since $L$ cannot be at infinite level, as remarked above, either $L$ is totally proper or we have $\bar{L}=U \cup S(L)$ as in (b) of (7.0). The leaves in $U$ have the same growth as $L$. If $L^{\prime}$ is a leaf of $S(L)$ at highest level, then $L^{\prime} \subset \delta U(4.1)$ and, by [C-C3, Lemma 4], $k$ strictly exceeds the degree of growth of $L^{\prime}$. Thus, $k$ exceeds the degree of growth of every leaf in $S(L)$. 
(7.3) Corollary. Let $L$ be a leaf of $\mathcal{F}$ having a proper side and suppose that $L$ is approached by a leaf $L^{\prime}$ with nonexponential growth. Then $L$ is totally proper, it has an element of contracting holonomy on whatever side is approached by $L^{\prime}$, and $H^{1}(L ; \mathbf{R}) \neq 0$.

Proof. The fact that $L$ is totally proper is contained in (7.0) and (5.0). The remaining assertions are proven exactly as in [P1, Theorem 5.4], using (3.8).

This result generalizes [C-C3, Theorem 2], giving a positive answer to the conjecture on p. 99 of that reference. It also generalizes a compact leaf theorem of Plante [P1, Theorem 8.1] that is closely related to the Reeb-Thurston stability theorem [Th], [R-S]. This suggests possible extensions of the stability theorem to leaves with a proper side (cf. [Di, Theorem 4]; but the proof, if not the assertion, is incorrect). The best such extensions will probably be to totally proper leaves. Here, using our present theory, we formulate an initial result in this direction.

(7.4) Corollary. Suppose that the leaves of $\mathcal{F}$ all have nonexponential growth and that $L$ is a proper leaf (necessarily totally proper) with $H^{1}(L ; \mathbf{R})=0$. Then $L$ has an open, $\mathcal{F}$-saturated neighborhood $U$ that is fibered over $S^{1}$ by $\mathscr{F} \mid U$.

Proof. By (7.3), $L$ cannot be approached on either side by any leaf. By (3.4), $L$ has an $\mathscr{F}$-saturated neighborhood $V \cong L \times(-1,1)$ with $L \times\{0\}=L$ and $L \times\{t\}$ a leaf of $\mathscr{F} \mid V$ for a (necessarily closed) set of values $t$ clustering at 0 from both sides. If there is a gap $V_{0}=L \times\left(t_{0}, t_{1}\right)$ in which no $L \times\{t\}$ is a leaf of $\mathscr{F}$, but $L \times\left\{t_{0}\right\}$ and $L \times\left\{t_{1}\right\}$ are leaves of $\mathscr{F}$, then $L \times\left\{t_{0}\right\}$ satisfies the same hypotheses as $L$, so (7.3) and (3.4) again apply to contradict the assumption on $V_{0}$. Thus, the union $W$ of all proper leaves homeomorphic to $L$ is an open set, smoothly fibered by $\mathscr{F} \mid W$ over some Hausdorff 1 -manifold without boundary.

Let $U$ be the component of $W$ containing $L$. The above fibration restricts to a fibering $p: U \rightarrow B$, where $B$ is a connected, Hausdorff 1-manifold without boundary. Thus $B \cong \mathbf{R}$ or $B \cong S^{1}$. If we can produce a smooth, closed transversal $\sigma \subset U$, then $p$ immerses $\sigma$ in $B$, so $B \cong S^{1}$.

If $\delta U=\varnothing$, then $U=M$ and again $B \cong S^{1}$, so we are done. Otherwise, let $L^{\prime} \subseteq \delta U$. If the holonomy of $L^{\prime}$ is not unbounded on a side bordering $U$, then (3.4) implies that $L^{\prime} \subset U$. Thus, on a side bordering $U, L^{\prime}$ is approached by a leaf of $\mathscr{F} \mid U$. In standard fashion, this allows us to construct a smooth, closed transversal $\sigma \subset U$, as desired.

\section{REFERENCES}

[A-S] L. V. Ahlfors and L. Sario, Riemann surfaces, Chapter 1, 86, Princeton Univ. Press, Princeton, N. J., 1960.

[B] W. M. Boothby, An introduction to differentiable manifolds and Riemannian geometry, Academic Press, New York, 1975.

[C-CO] J. Cantwell and L. Conlon, Leaves of finite classes, Notices Amer. Math. Soc. 25 (1978), A-146; Abstract 752-57-8.

[C-C1] _, Leaf prescriptions for closed 3-manifolds, Trans. Amer. Math. Soc. 236 (1978), 239-261.

[C-C2] __ Leaves with isolated ends in foliated 3-manifolds, Topology 16 (1977), 311-322.

[C-C3] _ Growth of leaves, Comment. Math. Helv. 53 (1978), 93-111. 
[C-C4]

[C-C5] Nonexponential leaves at finite level, preprint.

[De] A. Denjoy, Sur les courbes définies par les équations différentielles á la surface du tore, J. Math. Pures Appl. 11 (1932), 333-375.

[Di] P. Dippolito, Codimension one foliations of closed manifolds, Ann. of Math. 107 (1978), 403-453.

[Ha] A. Haefliger, Variétés feuilletees, Ann. Scuola Norm. Sup. Pisa (3) 16 (1962), 367-397.

[He] G. Hector, Leaves whose growth is neither exponential nor polynomial, Topology 16 (1977), 451-459.

[K] N. Kopell, Commuting diffeomorphisms, Proc. Sympos. Pure Math., vol. 14, Amer. Math. Soc., Providence, R. I., 1970, pp. 165-184.

[La] C. Lamoureux, Feuilles fermées et captage; applications, C. R. Acad. Sci. Paris Sér. A 277 (1973), 579-581.

[M-P] R. Moussu and F. Pelletier, Sur le théorème de Poincaré-Bendixson, Ann. Inst. Fourier Grenoble 24 (1974), 131-148.

[Ni] T. Nishimori, Isolated ends of open leaves of codimension-one foliations, Quart. J. Math. Oxford 26 (1975), 159-167.

[P1] J. F. Plante, Foliations with measure preserving holonomy, Ann. of Math. 102 (1975), 327-361.

[P2] _ A generalization of the Poincaré-Bendixson theorem for foliations of codimension one, Topology 12 (1973), 177-181.

[P3] , On the existence of exceptional minimal sets in foliations of codimension one, J. Differential Equations 15 (1974), 178-194.

[P4] (Measure preserving pseudogroups and a theorem of Sacksteder, Ann. Inst. Fourier Grenoble 25 (1975), 237-249.

[P5] __ Asymptotic properties of foliations, Comment. Math. Helv. 47 (1972), 449-456.

[R-S] G. Reeb and P. A. Schweitzer, Un théorème de Thurston établi au moyen de l'analyse non standard, in Differential Topology, Foliations, and Gelfand-Fuks Cohomology, Proceedings (Rio de Janeiro, 1976), Lecture Notes in Math. vol. 652, Springer-Verlag, New York, 1978, p. 138.

[Sa1] R. Sacksteder, On the existence of exceptional leaves in foliations of codimension one, Ann. Inst. Fourier Grenoble 15 (1965), 201-213.

[Sa2] __ Foliations and pseudogroups, Amer. J. Math. 87 (1965), 79-102.

[Sc] A. J. Schwartz, A generalization of a Poincaré-Bendixson theorem to closed two-dimensional manifolds, Amer. J. Math. 85 (1963), 453-458.

[Sch] P. A. Schweitzer, Counterexamples to the Seifert conjecture and opening closed leaves of foliations, Ann. of Math. 100 (1974), 386-400.

[So] J. Sondow, When is a manifold a leaf of some foliation?, Bull. Amer. Math. Soc. 81 (1975), $622-624$.

[S-S] R. Sacksteder and A. J. Schwartz, Limit sets of foliations, Ann. Inst. Fourier Grenoble 15 (1965), 201-214.

[Th] W. Thurston, A generalization of the Reeb stability theorem, Topology 13 (1974), 347-352.

[Ts] N. Tsuchiya, Leaves of finite depth, preprint.

Department of Mathematics, St. Louis University, St. Louis, Missouri 63103

Department of Mathematics, Washington University, St. Louis, Missouri 63130 